\title{
Measuring Performance with Common Weights: Network DEA
}

\author{
Adel Hatami-Marbini* \\ Department of Strategic Management and Marketing \\ Leicester Castle Business School \\ De Montfort University \\ Hugh Aston Building, The Gateway, Leicester LE1 9BH, UK \\ E-mail: adel.hatamimarbini@dmu.ac.uk
}

\author{
Saber Saati \\ Department of Mathematics \\ Tehran-North Branch, Islamic Azad University \\ P.O. Box 19585-936, Tehran, Iran \\ E-mail: $\underline{\text { s_saatim@iau-tnb.ac.ir }}$
}

\section{ACKNOWLEDGEMENT}

The authors would like to thank the anonymous reviewers and the associate editor for their insightful comments and suggestions.

* Corresponding author 


\title{
Measuring Performance with Common Weights: Network DEA
}

\begin{abstract}
In conventional data envelopment analysis (DEA), a production system has been seen as a black box for measuring the efficiency without any attention to what is happening inside the system. However, in practice, performance improvement often requires observing the internal structure of the producing system in order to find the sources of inefficiencies. In addition, weight flexibility as a key property of the multiplier DEA models allows a system to totally disregard an assessment factor, either input or output, from the evaluation process by assigning a value of zero or epsilon to its weight. This paper contributes to the existing literature by proposing a common-weights DEA model when the production system includes a number of interrelated processes. To this end, we propose an aggregate DEA model to calculate the most favourable common weights for determining the efficiency of all production systems and their processes at the same time. Our proposed aggregate model is not only linear for equitably evaluating the producing units on the same scale, but also enables us to deal with the mixed network structures. Furthermore, the network system is decomposed into a series system to build a relational network DEA model that emphasises separate relatedness. This greatly reduces the computational complexities for enormous volumes of data in many real applications and treat difficulties in network DEA models including the zero-value and fluctuating weights and multiple solutions. Managerially speaking, this paper aims to provide top management teams of a production system with an integrated framework to shape a better strategic decision process coupled with superior firm performance, which is to determine the sources of inefficiencies and ultimately take corrective actions over the long run. The proposed framework helps top managers make proper decisions in complex situations with a view of improving a firm's efficiency in all production divisions, which can be identified as a core competency leading to competitive advantages. In a context of performance management, our study is equipped with a simple numerical example and a case study of the non-life insurance companies to demonstrate the applicability of the proposed common-weights network model.
\end{abstract}

Keywords: Network structures; Data envelopment analysis; Common set of weights; Decomposition. 


\section{Introduction}

Data envelopment analysis (DEA) is a well-established methodology for measuring the relative efficiency of a set of peer entities called decision making units (DMUs) which convert inputs into multiple [1]. The basic DEA models are the CCR named after Charnes, Cooper, and Rhodes [1] and BCC models named after Banker, Charnes, and Cooper [2] to assess efficiency measures under the assumption of constant returns-to-scale (CRS) and variable returns-to-scale $(\mathrm{VRS})^{1}$, respectively. DEA as a nonparametric method builds on the engineering ratio concept by the use of the multiple-input multiple-output ratio rather than a single-input and singleoutput ratio for efficiency analysis. In the presence of multiple inputs and outputs, the technical efficiency measure of a DMU is defined as maximising the ratio of a weighted sum of outputs to a weighted sum of inputs such that this ratio always lies between zero and 1 .

\subsection{Common weights}

The multiplier DEA model allows full flexibility to assign the most favourable endogenous weights to the inputs and outputs as to compute the efficiency of a certain DMU under evaluation. However, with reference to Charnes et al. [3], we have need of further attention to control on weights in four situations: $(i)$ the results take no account of some inputs and/or outputs when their assigned weights are zero (or epsilon), (ii) the decision maker does not concur with the results, (iii) the relative importance of some inputs and/or outputs are determined by the decision maker, (iv) when the sum of numbers of inputs outputs is relatively larger than the number of the DMUs, the DEA model cannot succeed in discriminating between DMUs and one observes many efficient DMUs. Cook et al. [4] and Roll et al. [5] treated the total weight flexibility of the conventional DEA models by introducing a common set of weights (CSW). The aim of common-weights DEA model is to use a set of unique weights for evaluating all the DMUs. Roll et al. [5] obtained a CSW for those situations where the upper and lower bounds are imposed on weights. In their method, the weight variation of each factor first decreases and then the average of weights was defined to compute the efficiency scores of the various DMUs. Doyle and Green [6] considered the average of the optimal weights as a CSW where the input and output weights are derived from the conventional DEA model. Liu and Peng [7] sought one CSW as the most favourable for identifying the absolute efficiency for efficient DMUs as to provide a ranking order of a group of DMUs. Taking into account an

\footnotetext{
${ }^{1}$ CRS means the input fluctuation would result in the same proportional the output fluctuation, and VRS implies a disproportionate increase or decrease in outputs when inputs have uplifted.
} 
ideal virtual unit, Saati et al. [8] developed a two-phase CSW approach to evaluate the performance of Danish district heating plants. Hosseinzadeh Lotfi et al. [9] and HatamiMarbini et al. [10] introduced a multi-objective programming (MOP) problem to maximise the efficiency score of all DMUs using a CSW. As an extension of cross-weight evaluation, Wang and Chin [11] introduced the neutral DEA model to yield a CSW for all the DMUs without being aggressive or benevolent to the other DMUs.

\subsection{Network DEA}

In the existing common-weights DEA models, the researchers often ignore the internal structure of production process of a DMU and focus on a single process while taking account of the internal configuration of the DMUs instead of viewing them as black boxes is sine qua non for a detailed analysis of real-life problems. Generally speaking, a production process encompasses different interrelated processes where each one has its own exogenous inputs and final outputs along with intermediate measures that are produced and consumed within the system. This type of view of the interdependencies among evaluated DMUs seeks alternative DEA models which are known as network DEA (cf. [12]). The research studies of Färe and Grosskopf [12] and Färe and Grosskopf [13] are recognised as a start point for developing DEA network models in the literature. The DEA network models account for the impact of internal processes which are manifestly important in measuring the performance of DMUs. Although Wang et al. [14] disregarded the intermediate measures and computed an efficiency of each process independently, Seiford and Zhu [15] took the intermediate measures into account to obtain the efficiency of profitability and marketability processes of the top 55 US commercial banks. In line with a two-process configuration, Chen and Zhu [16] showed that Seiford and Zhu [15]'s models are not able to appropriately characterise the interpretation of the DEA frontier in the presence of intermediate measures. As a matter of fact, the intermediate measures can build potential conflicts between the two consecutive processes in a way that the second process may reduce its intermediate measures to increase the amount of efficiency while at the same time the increase in intermediate measures lead to improving the efficiency of the first process.

Network DEA models have been attracted much interest in the recent DEA literature. The network system in practice includes the series, parallel and mixed (general) structures such as electric power companies, hospitals and broadcasting companies [17]. The popularity and abundance of network DEA models encouraged Castelli et al. [18], Cook et al. [19], and Agrell and Hatami-Marbini [20], Kao [21] and Castelli and Pesenti [22] to provide the overarching 
surveys of the advanced applications and models. Kao [21] reviewed studies on network DEA and proposed two distinct classifications according to the structure of the network systems and the developed models. The first classification entails nine categories based on the models: independent, system distance measure, process distance measure, factor distance measure, slacks-based measure, ratio-form system efficiency, ratio-form process efficiency, game theoretic, and value-based. Focusing on the structures, the second classification is composed of seven categories: basic two-stage, general two-stage, series, parallel, mixed, hierarchical, and dynamic models. Castelli and Pesenti [22] as an extension of Castelli et al. [18] provided a classification of the existing network DEA models and their applications to different industries as presented in Figure 1.

\section{=====Insert Figure 1 here=====}

The two-stage DEA models are the most developed network structures where each DMU is composed of two connected processes and intermediate measures play the link role between the consecutive processes. Cook et al. [19] and Agrell and Hatami-Marbini [20] carried out surveys on the DEA models with two-stage network structures. Agrell and Hatami-Marbini [20] reviewed the state of the art of two-stage DEA models with a particular emphasis on performance analysis in supply chain management. By the use of the unified notations, Agrell and Hatami-Marbini [20] classified the pertinent models into the three groups; (i) two-stage processes, (ii) game theory, and (iii) bi-level programming.

The network DEA models take into consideration the interrelationship of the processes within the system to measure the efficiency of the system and those of the processes at the same time. However, based on the above-mentioned literature about network DEA, the mathematical interrelationship of the process efficiencies with the system efficiency has been rarely studied.

In case of two processes connected in series, Kao and Hwang [23] proposed a DEA model to measure the efficiencies of the system and two processes at once where the system is the product of those of the two processes. Kao [24] extended the interrelationship idea of efficiency to a relational network DEA model with the aim of measuring the efficiencies of the system and processes at one time. The author also transformed a network system into a series system by defining a number of dummy processes, in which each stage in the series includes a set of parallel processes. We believe that the results acquired from Kao [24] are more robust and discriminative along with being able to handle many real-world applications which have network production systems. Thereby, the main idea of this paper is to extend the method developed by Kao [24] by controlling the flexibility in the choice of endogenous weights. 
With the exception of Yang and Liu [25] the common-weights DEA as a popular tool for controlling the weight flexibility has not been well explored when the internal structure is known. Yang and Liu [25] proposed a multiple objectives network DEA model to evaluate banking and branch efficiency with the two-stage series structure in Taiwan. Their approach includes two limitations. First, although the authors used the fuzzy approach proposed by Zimmermann [26] to transfer the multiple objectives programming model into one objective programming model, the obtained model to find the common multipliers is non-linear. Second, their method is limited to a certain situation in which every DMU is composed of two processes in series.

\subsection{Contribution}

In this paper, we develop a new common-weights DEA model for the network structure with a sort of the interrelated processes, to measure the technical efficiency of each production systems and their processes. To this end, we adapt the common-weights DEA model proposed by Hosseinzadeh Lotfi et al. [9] to the network structure in a way that the efficiencies of all systems and their processes are maximised at the same time. Furthermore, the existence of multiple solutions that possibly occurs in Hosseinzadeh Lotfi et al. [9]'s method is treated to yield more reliable efficiencies. Another contribution is that the network system is decomposed into a series system to construct a relational network DEA model in the presence of a set of common weights. A numerical example and a case study of 24 non-life insurance companies are given to illustrate the procedure and applicability of the proposed common-weights network model.

\subsection{Organisation}

The rest of the paper is organised as follows: Section 2 reviews briefly the common-weights DEA model with an unknown interior structure and the multiplier network DEA model with a given interior structure. In Section 3, we present the details of our proposed method for measuring the efficiency of system and processes for each DMU. A simple numerical example is given in Section 4, and Section 5 presents a real-world problem of 24 non-life insurance companies for evaluating the efficiencies using the proposed model. Conclusions and further research are discussed in Section 6.

\section{Background}

In this section, we first concisely present a common-weights DEA approach proposed by Hosseinzadeh Lotfi et al. [9] for evaluating production units with an unknown interior structure. We then review the multiplier network DEA model developed by Kao [24], considering the 
interrelationship of the processes in the production system to measure the efficiency of the system and its processes at once.

\subsection{Finding a CSW using a multi-objective approach}

Assume that there are a set of $n$ DMUs for the purpose of evaluation in which each $\mathrm{DMU}_{j}$ $(j=1, \ldots, n)$ consumes $m$ different inputs, $x_{i j}(i=1, \ldots, m)$, to produces different outputs, $y_{r j}$ $(r=1, \ldots, s)$. The objective of the multiplier DEA model is to maximise the ratio of all outputs over all inputs for a given DMUo, i.e., $\frac{\sum_{r=1}^{S} u_{r} y_{r o}}{\sum_{i=1}^{m} v_{i} x_{i o}}$ where $u_{r}$ and $v_{i}$ are the $r$ th output and $i$ th input weights. To evaluate all $\mathrm{DMU}_{j}$ on an identical scale, the following multi-objective fractional programming (MOFP) problem is expressed $[9,15]$ :

$$
\max \left\{\frac{\sum_{r=1}^{s} u_{r} y_{r j}}{\sum_{i=1}^{m} v_{i} x_{i j}} \mid j=1, \cdots, n\right\}
$$

s.t.

$\frac{\sum_{r=1}^{S} u_{r} y_{r j}}{\sum_{i=1}^{m} v_{i} x_{i j}} \leq 1, \quad \forall j$

$$
u_{r}, v_{i} \geq \varepsilon, \quad \forall r, i \text {. }
$$

This involves finding values for $u_{r}$ and $v_{i}$, such that the efficiency measures of all DMUs are maximised at once, subject to the constraint that all efficiency measures must be less than or equal to one. Given that solving model (1) is not straightforward, it has a need of determining the compromise solution. In this regard, a scalarised approach is prevalent for solving multiobjective optimisation models in which multiple objectives is converted into a single-objective optimisation problem. By the focus on the scalarised approach, the utility method [27], goal programming [28], interactive method [29], and fuzzy method [26, 30] are widely used in the literature. Taking into account the goal programming for the sake of minimising the deviations from the goal of each DMU (100\% efficiency), Hosseinzadeh Lotfi et al. [9] proposed the following linear programming model with a single-objective function:

$$
\min \sum_{j=1}^{n} \varphi_{j}
$$

s.t.

$\sum_{r=1}^{s} u_{r} y_{r j}-\sum_{i=1}^{m} v_{i} x_{i j}+\varphi_{j}=0, \quad \forall j$

$\varphi_{j} \geq 0, \quad u_{r}, v_{i} \geq \varepsilon, \quad \forall j, r, i$, 
where $\varphi_{j}$ is the sum of negative and positive deviations from the goal of the $j$ th DMU. The efficiency score of $\mathrm{DMU}_{j}$ can be then calculated using $\frac{\sum_{r=1}^{s} u_{r}^{*} y_{r j}}{\sum_{i=1}^{m} v_{i}^{*} x_{i j}}$ where $u_{r}^{*}$ and $v_{i}^{*}$ are the optimal output and input weights for all units resulted from the above model. Since model (2) is seeking a compromise solution, the positive value of its optimal objective shows the total amount of the variation from getting $100 \%$ efficiency for all the DMUs. Accordingly, all DMUs turn out to be efficient if the optimal objective value of (2) is equal to zero, i.e., $\varphi_{j}=$ $0, j=1,2, \ldots, n$.

As a quite possible problem, when multiple solutions occur in model (2) the efficiency of a certain DMU may be variant that would lead to bias in performance evaluation. In this paper, we strive deal with this problem by determining a unique efficiency measure.

\subsection{Multiplier network DEA model}

Several researchers have probed into network structures by taking account of multiplier DEA models. Kao [24] discussed the network system in the series, parallel and mixed (general) structures. He demonstrated how the general network structure can be converted into a series system in which each stage in the series has a parallel structure. Analogous to Kao [24], we limit our focus to a network structure with three processes as depicted in Figure 2.

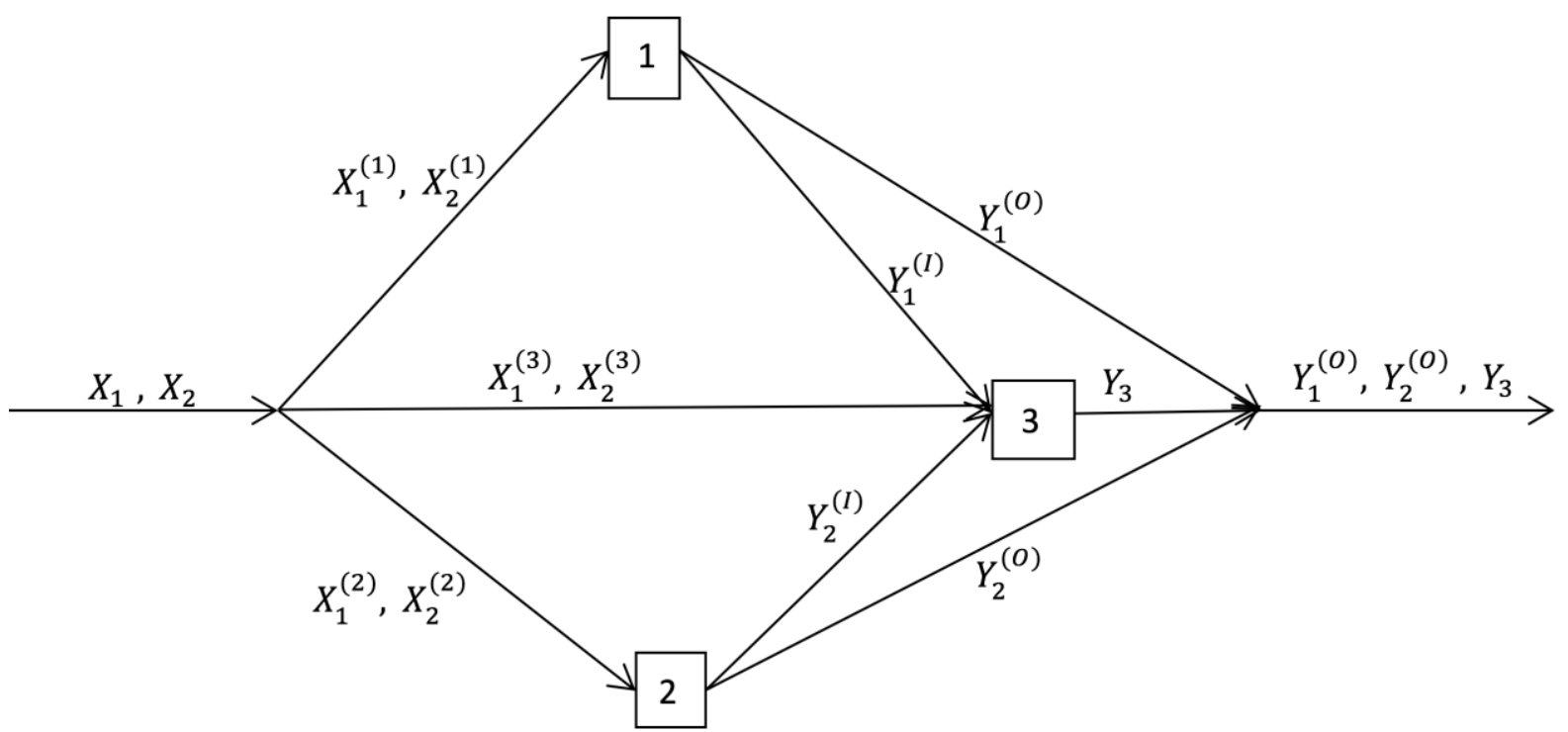

Figure 2. A network efficiency model

The system produces three different outputs $\left(Y_{1}, Y_{2}\right.$ and $\left.Y_{3}\right)$ by consumption of two different inputs $\left(X_{1}\right.$ and $\left.X_{2}\right)$. The portions of $X_{1}$ and $X_{2}$ are consumed by processes 1 and 2 (denoted by $X_{1}^{(1)}$ and $X_{2}^{(1)}$ for process 1 and $X_{1}^{(2)}$ and $X_{2}^{(2)}$ for process 2) to produce $Y_{1}$ for process 1 and $Y_{2}$ for process 2. Process 3 produces $Y_{3}$ by using the portions of $Y_{1}$ and $Y_{2}$ generated by processes 
1 and 2 (denoted by $Y_{1}^{(I)}$ and $Y_{2}^{(I)}$ ) as well as the remaining amount of $X_{1}$ and $X_{2}$ (denoted by $X_{1}^{(3)}$ and $\left.X_{2}^{(3)}\right)$. Assume that $x_{\mathrm{ij}}^{(k)}$ stands for the $i$ th input of process $k(k=1,2,3)$ and $x_{i j}^{(1)}+$ $x_{i j}^{(2)}+x_{i j}^{(3)}=x_{i j}, i=1,2, j=1, \cdots, n$ and let $y_{2 j}=\left(y_{2 j}^{(I)}+y_{2 j}^{(O)}\right), j=1, \cdots, n$ and $y_{1 j}=$ $\left(y_{1 j}^{(I)}+y_{1 j}^{(O)}\right), j=1, \cdots, n$. Let $Y_{r}^{(0)}$ and $Y_{r}^{(\mathrm{I})}, r=1,2$ denote the final outputs of the production system and the intermediate output consumed by process 3 , respectively. The following multiplier network DEA model associated with the aforesaid structure is formulated to compute the system efficiency of DMU。:

$$
\begin{aligned}
& E_{o}=\max u_{1} y_{1 o}^{(O)}+u_{2} y_{2 o}^{(O)}+u_{3} y_{3 o} \\
& \text { s.t. } \\
& v_{1} x_{1 o}+v_{2} x_{2 o}=1, \\
& \left(u_{1} y_{1 j}^{(O)}+u_{2} y_{2 j}^{(O)}+u_{3} y_{3 j}\right)-\left(v_{1} x_{1 j}+v_{2} x_{2 j}\right) \leq 0, \quad \forall j, \\
& u_{1} y_{1 j}-\left(v_{1} x_{1 j}^{(1)}+v_{2} x_{2 j}^{(1)}\right) \leq 0, \quad \forall j, \\
& u_{2} y_{2 j}-\left(v_{1} x_{1 j}^{(2)}+v_{2} x_{2 j}^{(2)}\right) \leq 0, \quad \forall j, \\
& u_{3} y_{3 j}-\left(v_{1} x_{1 j}^{(3)}+v_{2} x_{2 j}^{(3)}+u_{1} y_{1 j}^{(I)}+u_{2} y_{2 j}^{(I)}\right) \leq 0, \quad \forall j, \\
& u_{1}, u_{2}, u_{3}, v_{1}, v_{2} \geq \varepsilon,
\end{aligned}
$$

where $u_{r}(r=1,2,3)$ and $v_{i}(i=1,2)$ are the weights attached, respectively, to $r$ th output and $i$ th inputs. Constraint (3-1) bears on the system in which $\left(u_{1} y_{1 j}^{(O)}+u_{2} y_{2 j}^{(O)}+u_{3} y_{3 j}\right)$ and $\left(v_{1} x_{1 j}+v_{2} x_{2 j}\right)$ are the sum of weighted outputs and the sum of weighted inputs, respectively. Constraint (3-2) corresponds to process 1 in which $u_{1} y_{1 j}=u_{1}\left(y_{1 j}^{(I)}+y_{1 j}^{(O)}\right)$ and $\left(v_{1} x_{1 j}^{(1)}+\right.$ $\left.v_{2} x_{2 j}^{(1)}\right)$ are the weighted output and the sum of weighted inputs, respectively. Constraint (3-3) shows process 2 in which $u_{2} y_{2 j}=u_{2}\left(y_{2 j}^{(I)}+y_{2 j}^{(O)}\right)$ and $\left(v_{1} x_{1 j}^{(2)}+v_{2} x_{2 j}^{(2)}\right)$ are the sum of weighted output and the sum of weighted inputs, respectively. Constraint (3-4) corresponds to process 3 in which $u_{3} y_{3 j}$ is the weighted output and $\left(v_{1} x_{1 j}^{(3)}+v_{2} x_{2 j}^{(3)}+u_{1} y_{1 j}^{(I)}+u_{2} y_{2 j}^{(I)}\right)$ is the sum of weighted inputs. As one property of model (3), the weight is associated to each factor despite of the various processes, for instance, $x_{1 j}, x_{1 j}^{(1)}, x_{1 j}^{(2)}$ and $x_{1 j}^{(3)}$ are all weighted by $v_{1}$. The optimal weights $u_{r}^{*}(r=1,2,3)$ and $v_{i}^{*}(i=1,2)$ calculated from (3) yield the efficiencies of three processes for $\mathrm{DMU}_{\mathrm{o}}$ under evaluation as presented below: 


$$
\begin{aligned}
& E_{o}^{(1)}=u_{1}^{*} y_{1 o} /\left(v_{1}^{*} x_{1 o}^{(1)}+v_{2}^{*} x_{2 o}^{(1)}\right) \\
& E_{o}^{(2)}=u_{1}^{*} y_{2 o} /\left(v_{1}^{*} x_{1 o}^{(2)}+v_{2}^{*} x_{2 o}^{(2)}\right) \\
& E_{o}^{(3)}=u_{1}^{*} y_{3 o} /\left(v_{1}^{*} x_{1 o}^{(3)}+v_{2}^{*} x_{2 o}^{(3)}+u_{1}^{*} y_{1 o}^{(I)}+u_{2}^{*} y_{2 o}^{(I)}\right)
\end{aligned}
$$

The mixed network structure can be transformed into series and parallel structures for the purpose of efficiency decomposition. To this end, two dummy processes 4 and 5 are presented to arrive at a series system of two stages where the first stage entails a parallel system of three processes 1, 2 and 4, and the second stage encompasses a parallel system of two processes 3 and 5 (see Figure 3). Note that the variations imposed to the system by two dummy processes can be simply disregarded because the outputs and inputs of each one are identical. As a result of this re-configuration, the efficiency of stages 1 and 2, denoted by $E_{o}^{1}$ and $E_{o}^{2}$, can be calculated in a way that the system efficiency is the product of the efficiencies of two stages, i.e., $E_{o}=E_{o}^{1} \times E_{o}^{2}$ where $E_{o}^{1}=\left[u_{1}^{*} y_{1 o}+\left(v_{1}^{*} x_{1 o}^{(3)}+v_{2}^{*} x_{2 o}^{(3)}\right)+u_{2}^{*} y_{2 o}\right] /\left(v_{1}^{*} x_{1 o}+v_{2}^{*} x_{2 o}\right)$ and $E_{o}^{2}=\left(u_{1}^{*} y_{1 o}^{(o)}+u_{2}^{*} y_{2 o}^{(O)}+u_{3}^{*} y_{3 o}\right) /\left[u_{1}^{*} y_{1 o}+\left(v_{1}^{*} x_{1 o}^{(3)}+v_{2}^{*} x_{2 o}^{(3)}\right)+u_{2}^{*} y_{2 o}\right]$.

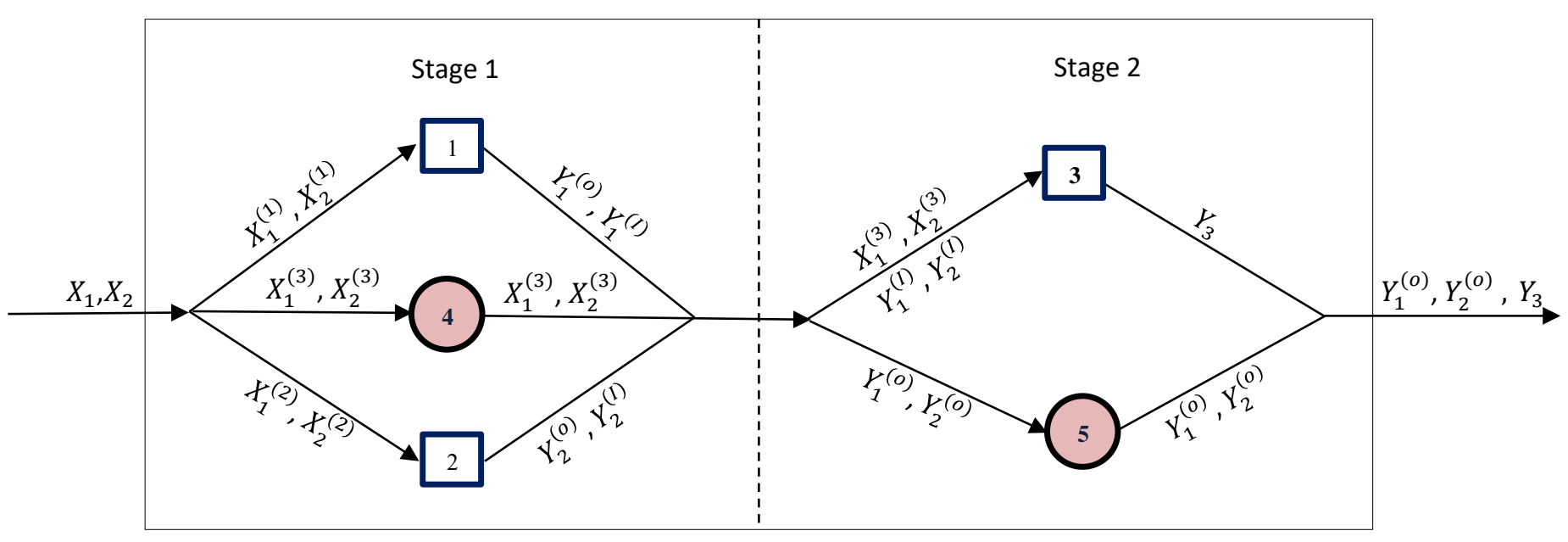

Figure 3. Two-stage configuration

At present, let us concentrate on two problems in Kao [24] 's approach. First, the efficiency measures of three processes are no longer unique in the case of multiple solutions for model (3) and second, the result of solving model (3) for each DMU leads to various set of weights that is most likely different from one DMU to another. In the next section, we propose a model that allows for determining the common weights in terms of the structure depicted in Figures 2 and 3.

\section{Common-weights network model}


In this section, we first present a common-weights network model with our emphasis on the whole production system and the idea is then extended to the processes in addition to the system.

\subsection{System orientation}

Referring to the mixed network structure proposed by Kao [24], this section adapts the common-weights approach of Hosseinzadeh Lotfi et al. [9] to determine the common weights for evaluating system and processes efficiencies. Let us follow the notations introduced in the previous section. We develop the following model to minimise the sum of the [nonnegative] deviations of all DMUs $\sum_{j=1}^{n} \varphi_{j}$ from their goals which are to be $100 \%$ efficient:

$$
\min \sum_{j=1}^{n} \varphi_{j}
$$

s.t.

$$
\begin{aligned}
& \left(u_{1} y_{1 j}^{(O)}+u_{2} y_{2 j}^{(O)}+u_{3} y_{3 j}\right)-\left(v_{1} x_{1 j}+v_{2} x_{2 j}\right)+\varphi_{j}=0, \quad \forall j \\
& u_{1} y_{1 j}-\left(v_{1} x_{1 j}^{(1)}+v_{2} x_{2 j}^{(1)}\right) \leq 0, \quad \forall j \\
& u_{2} y_{2 j}-\left(v_{1} x_{1 j}^{(2)}+v_{2} x_{2 j}^{(2)}\right) \leq 0, \quad \forall j \\
& u_{3} y_{3 j}-\left(v_{1} x_{1 j}^{(3)}+v_{2} x_{2 j}^{(3)}+u_{1} y_{1 j}^{(I)}+u_{2} y_{2 j}^{(I)}\right) \leq 0, \quad \forall j \\
& u_{1}, u_{2}, u_{3}, v_{1}, v_{2} \geq \varepsilon \\
& \varphi_{j} \geq 0, \quad \forall j
\end{aligned}
$$

Constraint (5-1) guarantees that the system efficiency of each DMU cannot exceed unity. Similarly, constraints (5-2), (5-3) and (5-4) guarantee that the efficiency of processes 1, 2 and 3 cannot exceed unity.

The occurrence of multiple solutions in model (5) deteriorates uniqueness of the efficiency measures for the system and processes and the associated ranking is resultantly not robust. To handle the problem, we propose a two-phase method, in which the first phase is to determine the optimal deviation of each DMU $\varphi_{j}^{*}$ using model (5) and the second phase is to solve the following model when $\varphi_{j}^{*}$ is preserved fixed:

$\max \left(u_{1} y_{1 o}^{(o)}+u_{2} y_{2 o}^{(o)}+u_{3} y_{3 o}\right) /\left(v_{1} x_{1 o}+v_{2} x_{2 o}\right)$

s.t.

$$
\begin{aligned}
& \left(u_{1} y_{1 j}^{(o)}+u_{2} y_{2 j}^{(O)}+u_{3} y_{3 j}\right)-\left(v_{1} x_{1 j}+v_{2} x_{2 j}\right)+\varphi_{j}^{*}=0, \quad \forall j, \\
& u_{1} y_{1 j}-\left(v_{1} x_{1 j}^{(1)}+v_{2} x_{2 j}^{(1)}\right) \leq 0, \quad \forall j,
\end{aligned}
$$


$u_{2} y_{2 j}-\left(v_{1} x_{1 j}^{(2)}+v_{2} x_{2 j}^{(2)}\right) \leq 0, \quad \forall j$,

$u_{3} y_{3 j}-\left(v_{1} x_{1 j}^{(3)}+v_{2} x_{2 j}^{(3)}+u_{1} y_{1 j}^{(I)}+u_{2} y_{2 j}^{(I)}\right) \leq 0, \quad \forall j$,

$u_{1}, u_{2}, u_{3}, v_{1}, v_{2} \geq \varepsilon$.

It is simple to discover that the above model is nonlinear. To linearize model (6), we first perform the change of variable as $v_{1} x_{1 o}+v_{2} x_{2 o}=\frac{1}{t}$ where $t$ represents a nonnegative variable. Thus, problem (6) is simplified to the following nonlinear model:

$\max t\left(u_{1} y_{1 o}^{(O)}+u_{2} y_{2 o}^{(o)}+u_{3} y_{3 o}\right)$

s.t.

$t\left(v_{1} x_{1 o}+v_{2} x_{2 o}\right)=1$,

$\left(u_{1} y_{1 j}^{(O)}+u_{2} y_{2 j}^{(O)}+u_{3} y_{3 j}\right)-\left(v_{1} x_{1 j}+v_{2} x_{2 j}\right)+\varphi_{j}^{*}=0, \quad \forall j$

$u_{1} y_{1 j}-\left(v_{1} x_{1 j}^{(1)}+v_{2} x_{2 j}^{(1)}\right) \leq 0, \quad \forall j$,

$u_{2} y_{2 j}-\left(v_{1} x_{1 j}^{(2)}+v_{2} x_{2 j}^{(2)}\right) \leq 0, \quad \forall j$,

$u_{3} y_{3 j}-\left(v_{1} x_{1 j}^{(3)}+v_{2} x_{2 j}^{(3)}+u_{1} y_{1 j}^{(I)}+u_{2} y_{2 j}^{(I)}\right) \leq 0, \quad \forall j$

$u_{1}, u_{2}, u_{3}, v_{1}, v_{2} \geq \varepsilon$,

$t \geq 0$.

We then arrive at the following linear programming model by multiplying both sides of constraints (7-1), (7-2), (7-3) and (7-4) by a nonnegative variable $t$ as well as substituting $t u_{r}(r=1,2,3)$ and $t v_{i}(i=1,2)$ with new variables $\mu_{r}$ and $\pi_{i}$, respectively:

$\max \left(\mu_{1} y_{1 o}^{(O)}+\mu_{2} y_{2 o}^{(O)}+\mu_{3} y_{3 o}\right)$

s.t.

$\left(\pi_{1} x_{1 o}+\pi_{2} x_{2 o}\right)=1$,

$\left(\mu_{1} y_{1 j}^{(o)}+\mu_{2} y_{2 j}^{(o)}+\mu_{3} y_{3 j}\right)-\left(\pi_{1} x_{1 j}+\pi_{2} x_{2 j}\right)+t \varphi_{j}^{*}=0, \quad \forall j$,

$\mu_{1} y_{1 j}-\left(\pi_{1} x_{1 j}^{(1)}+\pi_{2} x_{2 j}^{(1)}\right) \leq 0, \quad \forall j$,

$\mu_{2} y_{2 j}-\left(\pi_{1} x_{1 j}^{(2)}+\pi_{2} x_{2 j}^{(2)}\right) \leq 0, \quad \forall j$,

$\mu_{3} y_{3 j}-\left(\pi_{1} x_{1 j}^{(3)}+\pi_{2} x_{2 j}^{(3)}+\mu_{1} y_{1 j}^{(I)}+\mu_{2} y_{2 j}^{(I)}\right) \leq 0, \quad \forall j$,

$\mu_{1}, \mu_{2}, \mu_{3}, \pi_{1}, \pi_{2} \geq \varepsilon$,

$t \geq 0$. 
Lemma 1. Corresponding to each feasible solution of model (6), there exists a feasible solution to model (8) and vice versa.

Proof. Assume that $S_{1}$ and $S_{2}$ are the feasible regions of models (6) and (8), respectively. Let $(\pi, \mu) \in S_{1}$ be a feasible solution to model (6) where $\pi$ and $\mu$ are input and output weights. This feasible solution leads to $\pi_{1} x_{1 o}+\pi_{2} x_{2 o}=k$ and, consequently, $\frac{1}{k}(\pi, \mu, 1) \in S_{2}$ is a feasible solution to model (8) which enables to satisfy all the constraints of model (8). At present, assume that $(\pi, \mu, t) \in S_{2}$ is a feasible solution to model (8). Given that the scaler $t$ is nonnegative, $\frac{1}{t}(\pi, \mu)$ as a feasible solution satisfies all the constraints of model (6). Therefore, the feasibility of model (6) causes the feasibility of model (8) and vice versa.

Together with total weight flexibility in model (8), the solution $\left(\pi_{1}, \pi_{2}, \mu_{1}, \mu_{2}, \mu_{3}\right)=$ $(0,0,0,0,0)$ satisfies the constraints with the exception of the last constraint. Therefore, there exist many irrational or unreasonable weights in the optimal solution of model (8) whose values are very close to zero and these weights are highly dependent on the amount of epsilon (lower bound of weights) because of the finite precision in machine. To deal with the highly probable problem, we here adapt the idea introduced by Toloo [31] to get an optimal value of epsilon. The resulting model for computing the optimal epsilon is as follows:

$\max \varepsilon$

s. $\mathrm{t}$

$$
\begin{aligned}
& \pi_{1} x_{1 j}+\pi_{2} x_{2 j} \leq 1, \quad \forall j, \\
& \left(\mu_{1} y_{1 j}^{(O)}+\mu_{2} y_{2 j}^{(O)}+\mu_{3} y_{3 j}\right)-\left(\pi_{1} x_{1 j}+\pi_{2} x_{2 j}\right) \leq 0, \quad \forall j, \\
& \mu_{1} y_{1 j}-\left(\pi_{1} x_{1 j}^{(1)}+\pi_{2} x_{2 j}^{(1)}\right) \leq 0, \quad \forall j, \\
& \mu_{2} y_{2 j}-\left(\pi_{1} x_{1 j}^{(2)}+\pi_{2} x_{2 j}^{(2)}\right) \leq 0, \quad \forall j, \\
& \mu_{3} y_{3 j}-\left(\pi_{1} x_{1 j}^{(3)}+\pi_{2} x_{2 j}^{(3)}+\mu_{1} y_{1 j}^{(I)}+\mu_{2} y_{2 j}^{(I)}\right) \leq 0, \quad \forall j, \\
& \mu_{i}-\varepsilon \geq 0, \quad i=1,2,3, \\
& \pi_{r}-\varepsilon \geq 0, \quad r=1,2 .
\end{aligned}
$$

It must be emphasised that the optimal epsilon $\varepsilon^{*}$ obtained from the above model is utilised in models (5) and (8) as the lower bound of weights. 
Lemma 2. The optimal solution and optimal objective function values of models (6) and (8) are identical.

Proof. Assume that $\theta^{*}=\frac{\mu_{1}^{*} y_{10}^{(o)}+\mu_{2}^{*} y_{2 o}^{(o)}+\mu_{3}^{*} y_{3 o}}{\pi_{1}^{*} x_{1 o}+\pi_{2}^{*} x_{2 o}}$ is the optimal objective function value of model (6) where $\left(\pi^{*}, \mu^{*}\right) \in S_{1}$ is its optimal solution. Let $\pi_{1}^{*} x_{1 o}+\pi_{2}^{*} x_{2 o}=k^{*}$. Since $\frac{1}{k^{*}}\left(\pi^{*}, \mu^{*}, k^{*}\right)$ satisfies all the constraints of model $(8), \frac{1}{k^{*}}\left(\pi^{*}, \mu^{*}, k^{*}\right) \in S_{2}$. It is clear that the objective function value of model (8) turns into $\theta^{*}$ by using $\frac{1}{k^{*}}\left(\pi^{*}, \mu^{*}, k^{*}\right)$ as a feasible solution. It suffices to show that $\frac{1}{k^{*}}\left(\pi^{*}, \mu^{*}, k^{*}\right) \in S_{2}$ is an optimal solution to model (8).

Contrary to this, suppose that $(\tilde{\pi}, \tilde{\mu}, \tilde{t}) \in S_{2}$ is the optimal solution and $\tilde{\mu}_{1} y_{1 o}^{(o)}+\tilde{\mu}_{2} y_{2 o}^{(o)}+$ $\tilde{\mu}_{3} y_{3 o}>\theta^{*}$. Taking into account $\tilde{\pi}_{1} x_{1 o}+\tilde{\pi}_{2} x_{2 o}=1$, we have $\frac{\widetilde{\mu}_{1} y_{1 o}^{(o)}+\widetilde{\mu}_{2} y_{2 o}^{(o)}+\widetilde{\mu}_{3} y_{3 o}}{\widetilde{\pi}_{1} x_{1 o}+\widetilde{\pi}_{2} x_{2 o}}>\theta^{*}$. On the other hand, $(\tilde{\pi}, \tilde{\mu}, \tilde{t}) \in S_{2}$ leads to $\frac{1}{\tilde{t}}(\tilde{\pi}, \tilde{\mu}) \in S_{1}$ and the objective function value is $\theta=$ $\frac{\widetilde{\mu}_{1} y_{10}^{(o)}+\widetilde{\mu}_{2} y_{2 o}^{(o)}+\widetilde{\mu}_{3} y_{3 o}}{\widetilde{\pi}_{1} x_{1 o}+\widetilde{\pi}_{2} x_{2 o}}$. Resultantly, $\theta>\theta^{*}$ but this contradicts the optimality of $\left(\pi^{*}, \mu^{*}\right) \in S_{1}$. This result shows that $\frac{1}{k^{*}}\left(\pi^{*}, \mu^{*}, k^{*}\right) \in S_{2}$ and $\theta^{*}$ are the optimal solution and optimal objective function value of model (8). The proof is accomplished.

Let us now summarise the procedure introduced in this subsection for identifying the unique common set of weights. First, we obtain the optimal epsilon, $\varepsilon^{*}$, using model (9). Second, the optimal deviation of each DMU, $\varphi_{j}^{*}$, is calculated using model (5) where the lower bound of the weights is $\varepsilon^{*}$. The last step is to solve model (8) with respect to $\varepsilon^{*}$ and $\varphi_{j}^{*}$ in order to obtain the optimal [unique] common weights $\mu_{r}^{*}(r=1,2,3)$ and $\pi_{i}^{*}(i=1,2)$ that are deployed as the common base for evaluating the efficiency measure of the whole system and three processes as follows:

$$
\begin{aligned}
& E_{o}^{*}=\frac{\sum_{r=1}^{3} \mu_{r}^{*} y_{r j}}{\sum_{i=1}^{2} \pi_{i}^{*} x_{i j}} \\
& E_{o}^{(1)}=\mu_{1}^{*} y_{1 o} /\left(\pi_{1}^{*} x_{1 o}^{(1)}+\pi_{2}^{*} x_{2 o}^{(1)}\right) \\
& E_{o}^{(2)}=\mu_{1}^{*} y_{2 o} /\left(\pi_{1}^{*} x_{1 o}^{(2)}+\pi_{2}^{*} x_{2 o}^{(2)}\right) \\
& E_{o}^{(3)}=\mu_{1}^{*} y_{3 o} /\left(\pi_{1}^{*} x_{1 o}^{(3)}+\pi_{2}^{*} x_{2 o}^{(3)}+\mu_{1}^{*} y_{1 o}^{(I)}+\mu_{2}^{*} y_{2 o}^{(I)}\right)
\end{aligned}
$$

Efficiency decomposition can pinpoint the inefficient operation of the system in the form of a relational series of stages in order to provide further insight in the improvement process. We hence turn to Figure 3 and compute the efficiency of stages 1 and 2 using the common 
weights as $E_{o}^{1}=\left[\mu_{1}^{*} y_{1 o}+\left(\pi_{1}^{*} x_{1 o}^{(3)}+\pi_{2}^{*} x_{2 o}^{(3)}\right)+\mu_{2}^{*} y_{2 o}\right] /\left(\pi_{1}^{*} x_{1 o}+\pi_{2}^{*} x_{2 o}\right)$ and $E_{o}^{2}=$ $\left(\mu_{1}^{*} y_{1 o}^{(o)}+\mu_{2}^{*} y_{2 o}^{(o)}+\mu_{3}^{*} y_{3 o}\right) /\left[\mu_{1}^{*} y_{1 o}+\left(\pi_{1}^{*} x_{1 o}^{(3)}+\pi_{2}^{*} x_{2 o}^{(3)}\right)+\mu_{2}^{*} y_{2 o}\right] ;$ in which $E_{o}=E_{o}^{1} \times$ $E_{o}^{2}$

\subsection{Network orientation}

The main objective of earlier common-weights model is to achieve the goal of the production system, examining whether or not the system is efficiently performing. This subsection extends the common-weights model to include reaching the goals of all the processes as well as the goal of the system. We therefore develop the following common-weights network model to minimise the sum of the deviations of systems and processes from their goals at the same time:

$$
\min \sum_{j=1}^{n} \varphi_{j}+\sum_{j=1}^{J} \sum_{k=1}^{K=3} \gamma_{k j}
$$

s.t.

$$
\begin{aligned}
& \left(u_{1} y_{1 j}^{(O)}+u_{2} y_{2 j}^{(O)}+u_{3} y_{3 j}\right)-\left(v_{1} x_{1 j}+v_{2} x_{2 j}\right)+\varphi_{j}=0, \quad \forall j, \\
& u_{1} y_{1 j}-\left(v_{1} x_{1 j}^{(1)}+v_{2} x_{2 j}^{(1)}\right)+\gamma_{1 j}=0, \quad \forall j, \\
& u_{2} y_{2 j}-\left(v_{1} x_{1 j}^{(2)}+v_{2} x_{2 j}^{(2)}\right)+\gamma_{2 j}=0, \quad \forall j, \\
& y_{3 j}-\left(v_{1} x_{1 j}^{(3)}+v_{2} x_{2 j}^{(3)}+u_{1} y_{1 j}^{(I)}+u_{2} y_{2 j}^{(I)}\right)+\gamma_{3 j}=0, \quad \forall j, \\
& \varphi_{j}, \gamma_{k j} \geq 0, \quad \forall k, \forall j, \\
& u_{1}, u_{2}, u_{3}, v_{1}, v_{2} \geq \varepsilon^{*},
\end{aligned}
$$

where $\varphi_{j}$ represents the deviation of the $j$ th DMU from its goal, $\gamma_{k j}$ is the deviation of the $k$ th process associated to the $j$ th DMU from its goal, and $\varepsilon^{*}$ is the optimal lower bound for the weights obtained from model (9). The above model aims to maximise the efficiencies of all DMUs and their processes simultaneously by minimising their deviations from their goals (100\% efficient). Likewise, the optimal solutions of model (11) may not be unique which give rise to an unfair performance evaluation. Thus, a two-phase method is analogously presented to provide a unique common-weights. The first phase computes the optimal deviations associated to the systems and their processes, denoted by $\varphi_{j}^{*}$ and $\gamma_{k j}^{*}$, using model (11), and the second phase works out the following model by setting $\varphi_{j}^{*}$ and $\gamma_{k j}^{*}$ : 
$\max \frac{u_{1} y_{1 o}^{(o)}+u_{2} y_{2 o}^{(o)}+u_{3} y_{3 o}}{v_{1} x_{1 o}+v_{2} x_{2 o}}$

s.t.

$$
\begin{aligned}
& \left(u_{1} y_{1 j}^{(O)}+u_{2} y_{2 j}^{(O)}+u_{3} y_{3 j}\right)-\left(v_{1} x_{1 j}+v_{2} x_{2 j}\right)+\varphi_{j}^{*}=0, \quad \forall j, \\
& u_{1} y_{1 j}-\left(v_{1} x_{1 j}^{(1)}+v_{2} x_{2 j}^{(1)}\right)+\gamma_{1 j}^{*}=0, \quad \forall j, \\
& u_{2} y_{2 j}-\left(v_{1} x_{1 j}^{(2)}+v_{2} x_{2 j}^{(2)}\right)+\gamma_{2 j}^{*}=0, \quad \forall j, \\
& u_{3} y_{3 j}-\left(v_{1} x_{1 j}^{(3)}+v_{2} x_{2 j}^{(3)}+u_{1} y_{1 j}^{(I)}+u_{2} y_{2 j}^{(I)}\right)+\gamma_{3 j}^{*}=0, \quad \forall j, \\
& u_{1}, u_{2}, u_{3}, v_{1}, v_{2} \geq \varepsilon^{*},
\end{aligned}
$$

where $\varepsilon^{*}$ is the optimal lower bound for the weights calculated from model (9). Similarly, the above nonlinear programming model can be transformed into the following linear programming model:

$\max \mu_{1} y_{1 o}^{(o)}+\mu_{2} y_{2 o}^{(o)}+\mu_{3} y_{3 o}$

s.t.

$\pi_{1} x_{1 o}+\pi_{2} x_{2 o}=1$,

$\left(\mu_{1} y_{1 j}^{(O)}+\mu_{2} y_{2 j}^{(O)}+\mu_{3} y_{3 j}\right)-\left(\pi_{1} x_{1 j}+\pi_{2} x_{2 j}\right)+t \varphi_{j}^{*}=0, \quad \forall j$,

$\mu_{1} y_{1 j}-\left(\pi_{1} x_{1 j}^{(1)}+\pi_{2} x_{2 j}^{(1)}\right)+t \gamma_{1 j}^{*}=0, \quad \forall j$,

$\mu_{2} y_{2 j}-\left(\pi_{1} x_{1 j}^{(2)}+\pi x_{2 j}^{(2)}\right)+t \gamma_{2 j}^{*}=0, \quad \forall j$,

$\mu_{3} y_{3 j}-\left(\pi_{1} x_{1 j}^{(3)}+\pi_{2} x_{2 j}^{(3)}+\mu_{1} y_{1 j}^{(I)}+\mu_{2} y_{2 j}^{(I)}\right)+t \gamma_{3 j}^{*}=0, \quad \forall j$,

$\mu_{1}, \mu_{2}, \mu_{3}, \pi_{1}, \pi_{2} \geq \varepsilon^{*}$,

$t \geq 0$.

Let $\left(\pi_{r}^{*}, \mu_{i}^{*}\right), r=1,2 ; i=1,2,3$, be the optimal [unique] common weights calculated from the above model. The efficiency score of the system and three processes of the DMU under evaluation are therefore measured as follows:

$$
\begin{aligned}
& E_{o}^{*}=\frac{\sum_{r=1}^{3} \mu_{r}^{*} y_{r j}}{\sum_{i=1}^{2} \pi_{i}^{*} x_{i j}} \\
& E_{o}^{(1)}=\mu_{1}^{*} y_{1 o} /\left(\pi_{1}^{*} x_{1 o}^{(1)}+\pi_{2}^{*} x_{2 o}^{(1)}\right) \\
& E_{o}^{(2)}=\mu_{1}^{*} y_{2 o} /\left(\pi_{1}^{*} x_{1 o}^{(2)}+\pi_{2}^{*} x_{2 o}^{(2)}\right)
\end{aligned}
$$




$$
E_{o}^{(1)}=\mu_{1}^{*} y_{3 o} /\left(\pi_{1}^{*} x_{1 o}^{(3)}+\pi_{2}^{*} x_{2 o}^{(3)}+\mu_{1}^{*} y_{1 o}^{(I)}+\mu_{2}^{*} y_{2 o}^{(I)}\right)
$$

The raw efficiency scores do not necessarily consist of unit-valued efficiency. In addition, we can decompose the system efficiency in the form of a relational series of two stages to identify the inefficient operation of the system (see Figure 3). Taking into account $E_{o}=E_{o}^{1} \times E_{o}^{2}$ and the common weights $\mu_{r}^{*}(r=1,2,3)$ and $\pi_{i}^{*}(i=1,2)$, the efficiency of stages 1 and 2 are calculated as $E_{o}^{1}=\left[\mu_{1}^{*} y_{1 o}+\left(\pi_{1}^{*} x_{1 o}^{(3)}+\pi_{2}^{*} x_{2 o}^{(3)}\right)+u_{2}^{*} y_{2 o}\right] /\left(\pi_{1}^{*} x_{1 o}+\pi_{2}^{*} x_{2 o}\right)$ and $E_{o}^{2}=$ $\left(\mu_{1}^{*} y_{1 o}^{(o)}+\mu_{2}^{*} y_{2 o}^{(o)}+\mu_{3}^{*} y_{3 o}\right) /\left[\mu_{1}^{*} y_{1 o}+\left(\pi_{1}^{*} x_{1 o}^{(3)}+\pi_{2}^{*} x_{2 o}^{(3)}\right)+\mu_{2}^{*} y_{2 o}\right]$

Lemma 3. Corresponding to each feasible solution of model (11), there exists a feasible solution to model (13) and vice versa.

Proof. The proof is the same as the proof of Lemma 1 and is omitted.

Lemma 4. The optimal solution and optimal objective function values of models (11) and (13) are identical.

Proof. The proof is the same as the proof of Lemma 2 and is omitted.

As a final remark, other network structures can be adopted on the basis of the proposed method in this paper to evaluate the performance all the system and process efficiencies using the common weights.

\section{Numerical Example}

We illustrate the practical use of the proposed method by a simple numerical example taken from Kao [24]. Five DMUs \{A, B, C, D, E $\}$ are assumed to be evaluated with the similar network structure as depicted in Figure 2. Table 1 shows the inputs and outputs where the row "SYS" is associated with the data for the system. To provide a consistent comparison with the DEA models, let us first compute the optimal epsilon using model (9). As a result, the lower bound of the weights (epsilon) is 0.0344828 . If we consider the conventional black-box configuration, the efficiencies of five DMUs using the basic CCR model with optimal epsilon are $0.9266,0.8832,0.7377,1$ and 1, respectively. Therefore, DMUs $\{D, E\}$ and $\{A, B, C\}$ are classified into the efficient and inefficient sets, respectively. Since the internal processes of each DMU is known, the ignorance of the performance of individual processes leads to inappropriate results by all the means. 
To delve deeper into the production process, it has need to take account of the internal structure. The second column of Table 2 represents the system efficiencies of DMUs using model (3) proposed by Kao [24] where the epsilon is 0.0344828 .

Table 1. Five observations for Example 1.

\begin{tabular}{|l|c|c|c|c|c|c|c|c|}
\hline DMU & Process & $\boldsymbol{X}_{\mathbf{1}}$ & $\boldsymbol{X}_{\mathbf{2}}$ & $\boldsymbol{Y}_{\mathbf{1}}^{(\mathbf{o})}$ & $\boldsymbol{Y}_{\mathbf{1}}^{(\mathbf{I})}$ & $\boldsymbol{Y}_{\mathbf{2}}^{(\mathbf{o})}$ & $\boldsymbol{Y}_{\mathbf{2}}^{(\mathbf{I})}$ & $\boldsymbol{Y}_{\mathbf{3}}$ \\
\hline A & SYS & 11 & 14 & 2 & - & 2 & - & 1 \\
& 1 & 3 & 5 & 2 & 2 & - & - & - \\
& 2 & 4 & 3 & - & - & 2 & 1 & - \\
& 3 & 4 & 6 & - & 2 & - & 1 & 1 \\
\hline B & SYS & 7 & 7 & 1 & - & 1 & - & 1 \\
& 1 & 2 & 3 & 1 & 1 & - & - & - \\
& 2 & 2 & 1 & - & - & 1 & 1 & - \\
& 3 & 3 & 3 & - & 1 & - & 1 & 1 \\
\hline C & SYS & 11 & 14 & 1 & - & 1 & - & 2 \\
& 1 & 3 & 4 & 1 & 1 & - & - & - \\
& 2 & 5 & 3 & - & - & 1 & 1 & - \\
& 3 & 3 & 7 & - & 1 & - & 1 & 2 \\
\hline D & SYS & 14 & 14 & 2 & - & 3 & - & 1 \\
& 1 & 4 & 6 & 2 & 1 & - & - & - \\
& 2 & 5 & 5 & - & - & 3 & 1 & - \\
& 3 & 5 & 3 & - & 1 & - & 1 & 1 \\
\hline E & SYS & 14 & 15 & 3 & - & 2 & - & 3 \\
& 1 & 5 & 6 & 3 & 1 & - & - & - \\
& 2 & 5 & 4 & - & - & 2 & 2 & - \\
& 3 & 4 & 5 & - & 1 & - & 2 & 3 \\
\hline
\end{tabular}

Table 2. Efficiencies of systems, processes and stages calculated from Kao [24]'s method.

\begin{tabular}{|l|l|l|l|l|l|l|}
\hline DMU & SYS & Process 1 & Process 2 & Process 3 & Stage 1 & Stage 2 \\
\hline A & 0.4744 & 1 & 0.6613 & 0.3070 & 0.9013 & 0.5264 \\
\hline B & 0.5895 & 0.8188 & 1 & 0.5003 & 0.9286 & 0.6348 \\
\hline C & 0.5209 & 0.5618 & 0.3796 & 0.7204 & 0.6677 & 0.7801 \\
\hline D & 0.4706 & 0.5990 & 0.6069 & 0.4029 & 0.7174 & 0.6561 \\
\hline E & 0.7931 & 0.7273 & 0.6667 & 1 & 0.7931 & 1 \\
\hline
\end{tabular}

To compute the process efficiencies using Eq. (4), the optimal weights $\left\{v_{1}, v_{2}, u_{1}, u_{2}, u_{3}\right\}$ assigned to inputs and outputs for all the DMUs are required as listed in Table 3. It is observable that DMUs take different values for a specific weight, which might be in question for the managers. The three process efficiencies are shown in the $3^{\text {rd }}-5^{\text {th }}$ columns of Table 4 , and DMUs A, B and E are efficient in processes 1, 2 and 3, respectively, as highlighted in bold. In addition, the production structure can be reconfigured by introducing a dummy process (see Figure 3) to provide efficiency decomposition. This reconfiguration leads to stages 1 and 2 and their efficiencies for each DMU are listed in the last two columns of Table 4. Note that the 
system efficiency is equal to the product of the efficiencies of the two stages. For instance, the efficiencies of stage 1 and stage 2 for DMU C are 0.68 and 0.7549 and the product of these efficiencies is 0.5133 which is exactly the system efficiency for DMU C as indicated in italic in Table 4. From system standpoint, similar to the basic CCR model, DMU E is superior to other DMUs and one of the significant reasons is concerned with being efficient in stage 2 .

Table 3. Input and output weights calculated from Kao [24]'s method for five DMUs.

\begin{tabular}{|l|l|l|l|l|l|}
\hline DMU & $\boldsymbol{v}_{\mathbf{1}}$ & $\boldsymbol{v}_{\mathbf{2}}$ & $\boldsymbol{u}_{\mathbf{1}}$ & $\boldsymbol{u}_{\mathbf{2}}$ & $\boldsymbol{u}_{\mathbf{3}}$ \\
\hline $\mathrm{A}$ & 0.0470219 & 0.0344828 & 0.0783699 & 0.0642633 & 0.1891327 \\
\hline $\mathrm{B}$ & 0.0344828 & 0.1083744 & 0.1613300 & 0.0886700 & 0.3394910 \\
\hline $\mathrm{C}$ & 0.0470219 & 0.0344828 & 0.0783699 & 0.0642633 & 0.1891327 \\
\hline $\mathrm{D}$ & 0.0369458 & 0.0344828 & 0.0708128 & 0.0541872 & 0.1664614 \\
\hline E & 0.0344828 & 0.0344828 & 0.0689655 & 0.0517241 & 0.1609195 \\
\hline
\end{tabular}

At present, we utilise the proposed model (13) with the optimal epsilon 0.0344828 for the purpose of computing the common weights in terms of the network structure, and the results for $\left\{v_{1}, v_{2}, u_{1}, u_{2}, u_{3}\right\}$ are $\{0.0344828,0.0344828,0.0689655,0.0517241,0.1609195\}$. A remark that should be made is that the two inputs have the similar weight, which is 0.0344828 . Accordingly, the efficiencies of the system and three processes for each DMU are calculated using Eq. (14) as reported in $2^{\text {nd }}-5^{\text {th }}$ columns of Table 4.

Table 4. Efficiencies of systems, processes and stages calculated from the proposed method.

\begin{tabular}{|l|l|l|l|l|l|l|}
\hline DMU & SYS & Process 1 & Process 2 & Process 3 & Stage 1 & Stage 2 \\
\hline A & 0.4667 & $\mathbf{1}$ & 0.6429 & 0.3011 & 0.9000 & 0.5185 \\
\hline B & 0.5833 & 0.8000 & $\mathbf{1}$ & 0.4912 & 0.9286 & 0.6282 \\
\hline C & 0.5133 & 0.5714 & 0.3750 & 0.6914 & 0.6800 & 0.7549 \\
\hline D & 0.4702 & 0.6000 & 0.6000 & 0.4058 & 0.7143 & 0.6583 \\
\hline E & 0.7931 & 0.7273 & 0.6667 & $\mathbf{1}$ & 0.7931 & 1 \\
\hline
\end{tabular}

The series-structure of the original network system including two stages can be also represented to yield the different decomposition for the purpose of showcasing the interrelationship of the component processes. The last two columns of Table 4 indicate the efficiencies of stages 1 and 2. A point worth stating is that the system efficiency is the product of the efficiencies of two stages. For instance, the efficiencies of stages 1 and 2 of DMU B are 0.9286 and 0.6282 , respectively, and the product of these two values is equal to 0.5833 , which is the efficiency of the system. Even though our proposed method yields the common base for evaluating the 
efficiencies, it is a need to explore whether the system and process efficiencies appropriately represent the performance of DMUs. In this regard, we make a comparison between our results reported in Table 4 and the method proposed by Kao [24]. From the system viewpoint, the resulting efficiencies are $\{0.4667,0.5833,0.5133,0.4702,0.7931\}$ and $\{0.4744,0.5895$, $0.5209,0.4706,0.7931\}$ that are almost identical. If one takes into account the ranking order stemmed from both models, the common-weight method causes to swap the positions of DMUs A and D. From the process and stage viewpoints, the results in Tables 3 and 4 show that the corresponding efficiencies are quite identical. Generally speaking, the efficiency scores obtained from our method is not exceeded those calculated from Kao [24] for the majority of cases. Interestingly, considering the optimal epsilon as a lower bound for the weights in the basic CCR and Kao [24]'s models in lieu of an arbitrary small non-Archimedean number gives rise to improving the discriminatory power of the models. In particular, DMU B with the nonoptimal epsilon is efficient in the basic CCR model as reported in Kao [24] while it turns out to be inefficient and has the efficiency score of 0.9266 in the presence of the optimal epsilon. Furthermore, the efficiencies of system, three processes and stages for units with the optimal epsilon does not exceed those calculated with the non-optimal epsilon.

\section{An application}

The nonparametric approach to efficiency evaluation has observed many extensive applications in the insurance industry (e.g., $[32,23])$. To illustrate the applicability of our common-weights network DEA model in a real-world problem, we perform an empirical application for the nonlife insurance companies. Particularly, we use a data set with 24 non-life insurance companies in Taiwan initiated by Hwang and Kao [23] as a two-stage problem in which insurance service and capital investment are the first and second stages, respectively. The system includes two inputs, i.e., Insurance expenses (X1) and Investment expenses (X2), two intermediate measures, i.e., Direct written premiums (Z1) and Reinsurance premiums (Z2), and two outputs, i.e., Underwriting profit (Y1) and Investment profit (Y2). Hwang and Kao [23] measured the efficiency of each process of insurance companies independently. Later, Kao and Hwang [23] took a relational network DEA model into account to measure the efficiencies of the two processes and the system. A closer view of Kao and Hwang [23]'s approach reveals that the first stage uses $\mathrm{X} 1$ and $\mathrm{X} 2$ to generate $\mathrm{Z} 1$ and $\mathrm{Z} 2$ which become the inputs to the second stage (referred to as intermediate measures) and the second stage then uses these intermediate measures to produce Y1 and Y2. The network structure of the non-life insurance operation 
system is modified by Kao [24] in a way that \{Insurance expenses (X1), Underwriting profit $(\mathrm{Y} 1)\}$ and $\{$ Investment expenses (X2) and Investment profit (Y2)\} are concerned with insurance service and capital investment processes, respectively, and the two processes are interrelated via $\{$ Direct written premiums (Z1) and Reinsurance premiums (Z2)\}, as depicted in Figure 4. The inputs, outputs and intermediate measures used in Kao [24] are reported in Table 5.

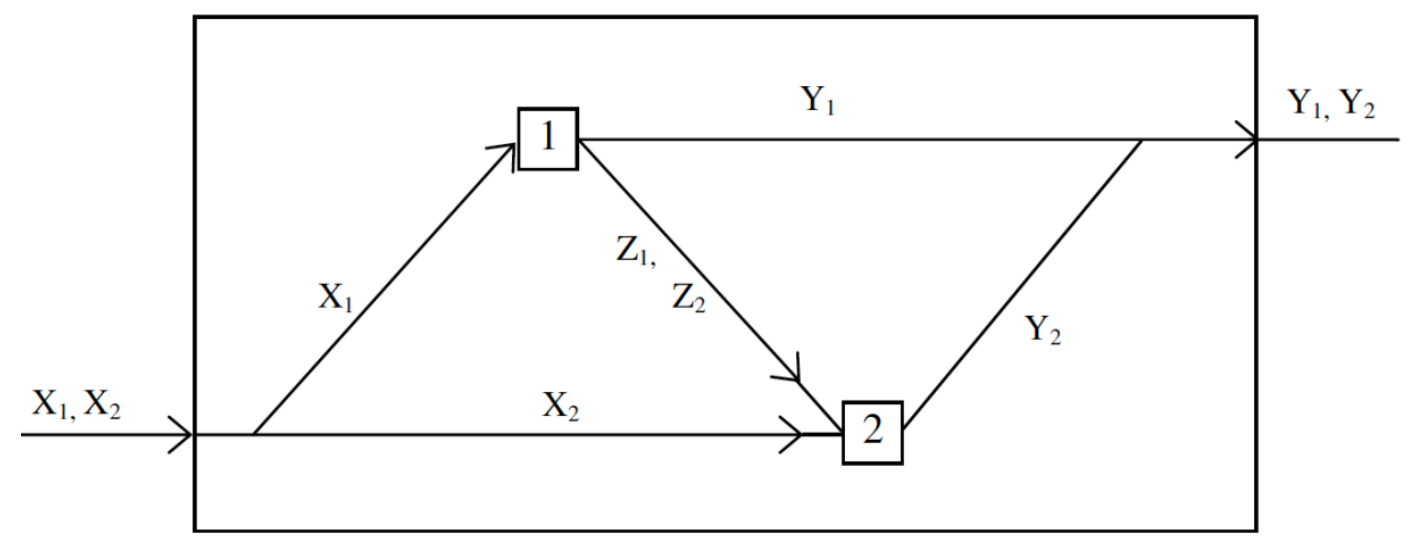

Figure 4. A network structure used in the non-life insurance operation system

The optimal epsilon as a lower bound of the weights is $1.04573 \mathrm{e}-08$ by adopting the proposed model in this study. Regardless the internal structure, the $2^{\text {nd }}$ column of Table 6 presents the efficiencies of 24 the non-life insurance companies calculated from the basic CCR model in the case of setting $\varepsilon=1.04573 \mathrm{e}-08$. Given the optimal epsilon, the system and processes efficiencies of companies are obtained using Kao [24]'s method as listed in $2^{\text {nd }}-4^{\text {th }}$ columns of Table 6. Note that the rank of each company is presented in parentheses in Table 6. In addition, the optimal weights $\left\{\mu_{1}, \mu_{2}, w_{1}, w_{2}, \pi_{1}, \pi_{2}\right\}$ allocated to inputs, intermediate measures and outputs for all the DMUs are reported in Table 7. By introducing two dummy processes, 3 and 4, the system efficiency is decomposed into two subsequent stages as shown in Figure 5. The last two columns of Table 6 report the efficiencies of the two stages.

Contrary to the individual efficiency model with endogenous weights proposed by Kao [24], the aggregate evaluation is proposed in this research by computing the set of non-zero common weights with the aim of achieving the goal of all the production systems (i.e., 100\% efficient) at one time. We therefore adopt our proposed model to the data sets listed in Table 4 in which non-Archimedean epsilon is set to be $1.04573 \mathrm{e}-08$. The resulting common weights $\left\{\mu_{1}, \mu_{2}, w_{1}, w_{2}, \pi_{1}, \pi_{2}\right\}$ are $\{2.33 \mathrm{e}-7,1.05 \mathrm{e}-8,1.05 \mathrm{e}-8,1.05 \mathrm{e}-8,1.05 \mathrm{e}-8,1.03 \mathrm{e}-7\}$, which are deployed to obtain system and two processes efficiencies as reported in Table 8. Compellingly, the minimum value of weights for each factor (see the last column of Table 7) and the resultant 
common weights are identical and that is why the common weights are very small. Furthermore, the efficiency decomposition as the product of the efficiencies of the two stages is given in the last two columns of Table 8 .

Table 5. The inputs, outputs and intermediate measures of the 24 non-life insurance companies.

\begin{tabular}{|c|c|c|c|c|c|c|c|}
\hline No. & Company & X1 & $\mathrm{X} 2$ & $\mathbf{Z 1}$ & $\mathbf{Z 2}$ & Y1 & Y2 \\
\hline 1 & Taiwan Fire & 673,512 & $1,178,744$ & $7,451,757$ & 856,735 & 984,143 & 681,687 \\
\hline 2 & Chung Kuo & $1,352,755$ & $1,381,822$ & $10,020,274$ & $1,812,894$ & $1,228,502$ & 834,754 \\
\hline 3 & Tai Ping & 592,790 & $1,177,494$ & $4,776,548$ & 560,244 & 293,613 & 658,428 \\
\hline 4 & China Mariners & 594,259 & 601,320 & $3,174,851$ & 371,863 & 248,709 & 177,331 \\
\hline 5 & Fubon & $3,531,614$ & $6,699,063$ & $37,392,862$ & $1,753,794$ & $7,851,229$ & $3,925,272$ \\
\hline 6 & Zurich & 668,363 & $2,627,707$ & $9,747,908$ & 952,326 & $1,713,598$ & 415,058 \\
\hline 7 & Taian & $1,443,100$ & $1,942,833$ & $10,685,457$ & 643,412 & $2,239,593$ & 439,039 \\
\hline 8 & Ming Tai & $1,873,530$ & $3,789,001$ & $17,267,266$ & $1,134,600$ & $3,899,530$ & 622,868 \\
\hline 9 & Central & 950,432 & $1,567,746$ & $11,473,162$ & 546,337 & $1,043,778$ & 264,098 \\
\hline 10 & The First & $1,298,470$ & $1,303,249$ & $8,210,389$ & 504,528 & $1,697,941$ & 554,806 \\
\hline 11 & Kuo Hua & 672,414 & $1,962,448$ & $7,222,378$ & 643,178 & $1,486,014$ & 18,259 \\
\hline 12 & Union & 650,952 & $2,592,790$ & $9,434,406$ & $1,118,489$ & $1,574,191$ & 909,295 \\
\hline 13 & Shingkong & $1,368,802$ & $2,609,941$ & $13,921,464$ & 811,343 & $3,609,236$ & 223,047 \\
\hline 14 & South China & 988,888 & $1,396,002$ & $7,396,396$ & 465,509 & $1,401,200$ & 332,283 \\
\hline 15 & Cathay Century & 651,063 & $2,184,944$ & $10,422,297$ & 749,893 & $3,355,197$ & 555,482 \\
\hline 16 & Allianz President & 415,071 & $1,211,716$ & $5,606,013$ & 402,881 & 854,054 & 197,947 \\
\hline 17 & Newa & $1,085,019$ & $1,453,797$ & $7,695,461$ & 342,489 & $3,144,484$ & 371,984 \\
\hline 18 & $\mathrm{AIU}$ & 547,997 & 757,515 & $3,631,484$ & 995,620 & 692,731 & 163,927 \\
\hline 19 & North America & 182,338 & 159,422 & $1,141,950$ & 483,291 & 519,121 & 46,857 \\
\hline 20 & Federal & 53,518 & 145,442 & 316,829 & 131,920 & 355,624 & 26,537 \\
\hline 21 & Royal \& Sunalliance & 26,224 & 84,171 & 225,888 & 40,542 & 51,950 & 6491 \\
\hline 22 & Asia & 10,502 & 15,993 & 52,063 & 14,574 & 82,141 & 4181 \\
\hline 23 & $\mathrm{AXA}$ & 28,408 & 54,693 & 245,910 & 49,864 & 0.1 & 18,980 \\
\hline 24 & Mitsui Sumitomo & 235,094 & 163,297 & 476,419 & 644,816 & 142,370 & 16,976 \\
\hline \multicolumn{2}{|c|}{ Mean } & 828,963 & $1,544,215$ & $7,832,893$ & 667,964 & $1,602,873$ & 477,733 \\
\hline
\end{tabular}

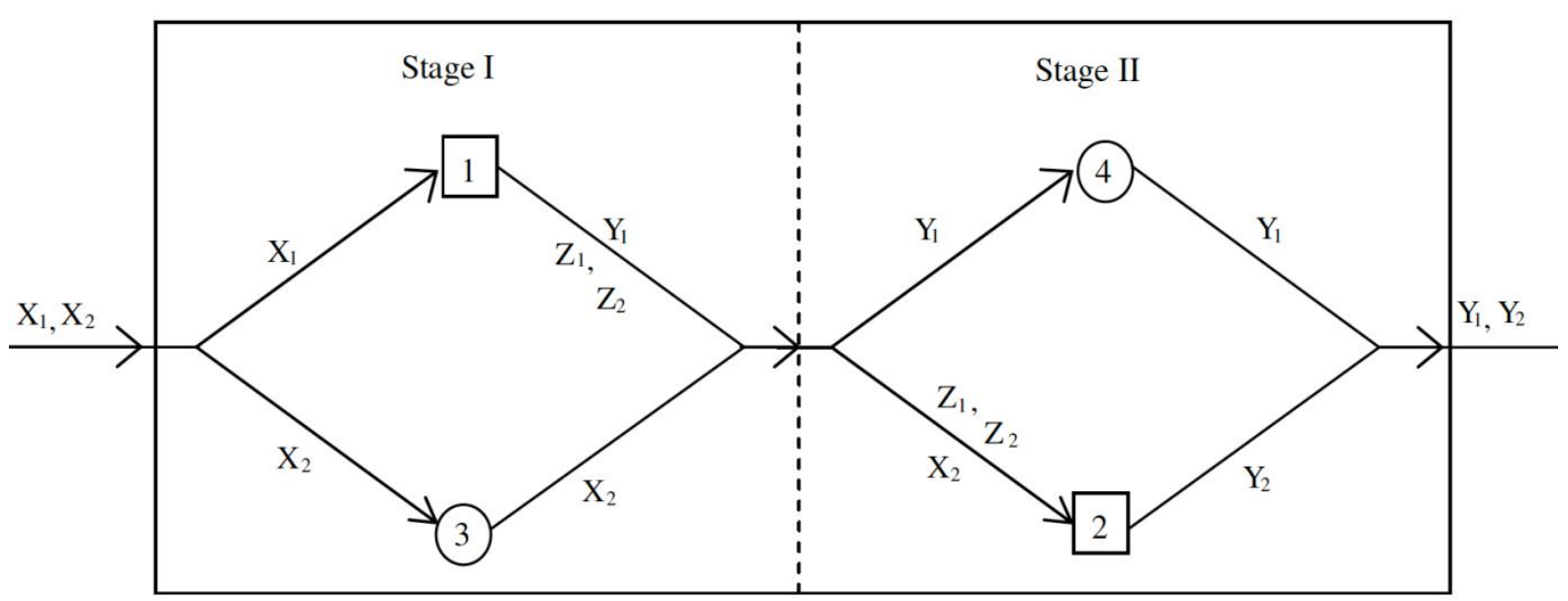

Figure 5. Two-stage structure for the non-life insurance operation system 
Table 6. Efficiencies of the basic CCR, system, processes and stages calculated from Kao [24]'s method for the 24 non-life insurance companies.

\begin{tabular}{|c|c|c|c|c|c|c|}
\hline No. & CCR & SYS & Process 1 & Process 2 & Stage 1 & Stage 2 \\
\hline 1 & $0.980(5)$ & $0.913(2)$ & $0.618(8)$ & $0.971(2)$ & $0.940(7)$ & $0.972(4)$ \\
\hline 2 & $1 \quad$ (1) & $0.805(5)$ & $0.433(17)$ & $0.981(1)$ & $0.821(14)$ & $0.981(3)$ \\
\hline 3 & $0.978(6)$ & $0.894(3)$ & $0.426(18)$ & $0.971(2)$ & $0.921(9)$ & $0.971(5)$ \\
\hline 4 & $0.488(14)$ & $0.450(12)$ & $0.306(23)$ & $0.501(8)$ & $0.896(11)$ & $0.503(18)$ \\
\hline 5 & $1 \quad$ (1) & $0.599(8)$ & $0.596(10)$ & 0.883 (4) & 0.667 (19) & $0.898(6)$ \\
\hline 6 & $0.590(13)$ & $0.403(14)$ & $0.736(6)$ & $0.322(17)$ & $0.743(18)$ & $0.543(15)$ \\
\hline 7 & $0.468(17)$ & $0.325(17)$ & $0.421(19)$ & $0.386(15)$ & $0.805(16)$ & $0.404(21)$ \\
\hline 8 & 0.415 (19) & $0.293(20)$ & 0.479 (14) & $0.275(20)$ & $0.500(21)$ & $0.586(13)$ \\
\hline 9 & $0.327(22)$ & $0.262(23)$ & $0.616(9)$ & $0.278(19)$ & $0.915(10)$ & $0.287(23)$ \\
\hline 10 & $0.776(10)$ & $0.582(9)$ & $0.359(21)$ & $0.716(5)$ & $0.806(15)$ & $0.722(9)$ \\
\hline 11 & $0.279(23)$ & $0.266(22)$ & $0.354(22)$ & 0.018 (24) & $0.367(24)$ & $0.725(8)$ \\
\hline 12 & $1 \quad$ (1) & $0.711(7)$ & $1 \quad$ (1) & $0.694(5)$ & $1 \quad$ (1) & $0.711(10)$ \\
\hline 13 & $0.352(20)$ & $0.320(18)$ & $0.464(15)$ & $0.127(23)$ & $0.479(22)$ & $0.669(11)$ \\
\hline 14 & $0.469(16)$ & $0.362(15)$ & $0.420(20)$ & $0.408(13)$ & $0.866(12)$ & $0.418(20)$ \\
\hline 15 & $0.975(7)$ & $0.729(6)$ & $1 \quad$ (1) & $0.394(14)$ & (1) & $0.729(7)$ \\
\hline 16 & $0.472(15)$ & $0.320(19)$ & $0.621(7)$ & $0.287(18)$ & $0.626(20)$ & $0.512(17)$ \\
\hline 17 & $0.634(11)$ & $0.420(13)$ & $0.503(12)$ & $0.440(12)$ & $0.786(17)$ & $0.535(16)$ \\
\hline 18 & $0.427(18)$ & $0.345(16)$ & $0.481(13)$ & $0.366(16)$ & $0.931(8)$ & $0.371(22)$ \\
\hline 19 & $0.821(9)$ & 0.480 & $0.538(11)$ & 0.485 (10) & $0.966(5)$ & 0.497 (19) \\
\hline 20 & $0.935(8)$ & $0.848(4)$ & $0.851(4)$ & $0.442(11)$ & $0.851(13)$ & $0.996(2)$ \\
\hline 21 & $0.333(21)$ & $0.268(21)$ & $0.451(16)$ & $0.208(21)$ & $0.451(23)$ & $0.593(12)$ \\
\hline 22 & $1 \quad$ (1) & $1 \quad(1)$ & $1 \quad$ (1) & $0.501(8)$ & (1) & (1) \\
\hline 23 & $0.599(12)$ & $0.579(10)$ & $0.800(5)$ & $0.585(7)$ & $0.991(4)$ & $0.585(14)$ \\
\hline 24 & $0.257(24)$ & $0.167(24)$ & $0.241(24)$ & $0.173(22)$ & $0.958(6)$ & $0.174(24)$ \\
\hline
\end{tabular}

Table 7. Input and output weights of Kao [24]'s method for the 24 non-life insurance companies.

\begin{tabular}{|l|c|c|c|c|c|c|}
\hline No. & $\mu_{1}$ & $\mu_{2}$ & $w_{1}$ & $w_{2}$ & $\pi_{1}$ & $\pi_{2}$ \\
\hline 1 & $2.33 \mathrm{e}-07$ & $7.15 \mathrm{e}-07$ & $1.05 \mathrm{e}-08$ & $1.05 \mathrm{e}-08$ & $1.05 \mathrm{e}-08$ & $1.32 \mathrm{e}-06$ \\
\hline 2 & $2.33 \mathrm{e}-07$ & $4.95 \mathrm{e}-07$ & $1.05 \mathrm{e}-08$ & $1.05 \mathrm{e}-08$ & $1.05 \mathrm{e}-08$ & $9.50 \mathrm{e}-07$ \\
\hline 3 & $2.33 \mathrm{e}-07$ & $7.32 \mathrm{e}-07$ & $1.05 \mathrm{e}-08$ & $1.05 \mathrm{e}-08$ & $1.05 \mathrm{e}-08$ & $1.35 \mathrm{e}-06$ \\
\hline 4 & $2.52 \mathrm{e}-07$ & $1.41 \mathrm{e}-06$ & $1.05 \mathrm{e}-08$ & $2.70 \mathrm{e}-08$ & $1.05 \mathrm{e}-08$ & $2.52 \mathrm{e}-06$ \\
\hline 5 & $2.33 \mathrm{e}-07$ & $2.63 \mathrm{e}-08$ & $1.05 \mathrm{e}-08$ & $1.05 \mathrm{e}-08$ & $1.05 \mathrm{e}-08$ & $1.32 \mathrm{e}-07$ \\
\hline 6 & $1.46 \mathrm{e}-06$ & $1.05 \mathrm{e}-08$ & $3.67 \mathrm{e}-08$ & $1.21 \mathrm{e}-07$ & $1.41 \mathrm{e}-07$ & $3.88 \mathrm{e}-07$ \\
\hline
\end{tabular}




\begin{tabular}{|l|l|l|l|l|l|l|}
\hline 7 & $2.33 \mathrm{e}-07$ & $3.41 \mathrm{e}-07$ & $1.05 \mathrm{e}-08$ & $1.05 \mathrm{e}-08$ & $1.05 \mathrm{e}-08$ & $6.87 \mathrm{e}-07$ \\
\hline 8 & $5.13 \mathrm{e}-07$ & $1.05 \mathrm{e}-08$ & $1.35 \mathrm{e}-08$ & $1.05 \mathrm{e}-08$ & $5.51 \mathrm{e}-08$ & $1.26 \mathrm{e}-07$ \\
\hline 9 & $2.33 \mathrm{e}-07$ & $4.96 \mathrm{e}-07$ & $1.05 \mathrm{e}-08$ & $1.05 \mathrm{e}-08$ & $1.05 \mathrm{e}-08$ & $9.51 \mathrm{e}-07$ \\
\hline 10 & $2.33 \mathrm{e}-07$ & $5.35 \mathrm{e}-07$ & $1.05 \mathrm{e}-08$ & $1.05 \mathrm{e}-08$ & $1.05 \mathrm{e}-08$ & $1.02 \mathrm{e}-06$ \\
\hline 11 & $1.46 \mathrm{e}-06$ & $1.05 \mathrm{e}-08$ & $1.05 \mathrm{e}-08$ & $1.05 \mathrm{e}-08$ & $1.78 \mathrm{e}-07$ & $1.03 \mathrm{e}-07$ \\
\hline 12 & $1.49 \mathrm{e}-06$ & $1.05 \mathrm{e}-08$ & $5.85 \mathrm{e}-08$ & $3.27 \mathrm{e}-07$ & $3.53 \mathrm{e}-08$ & $7.21 \mathrm{e}-07$ \\
\hline 13 & $7.11 \mathrm{e}-07$ & $1.05 \mathrm{e}-08$ & $1.05 \mathrm{e}-08$ & $1.05 \mathrm{e}-08$ & $8.24 \mathrm{e}-08$ & $1.03 \mathrm{e}-07$ \\
\hline 14 & $2.33 \mathrm{e}-07$ & $5.51 \mathrm{e}-07$ & $1.05 \mathrm{e}-08$ & $1.05 \mathrm{e}-08$ & $1.05 \mathrm{e}-08$ & $1.04 \mathrm{e}-06$ \\
\hline 15 & $1.50 \mathrm{e}-06$ & $1.05 \mathrm{e}-08$ & $4.00 \mathrm{e}-08$ & $1.05 \mathrm{e}-08$ & $1.65 \mathrm{e}-07$ & $3.18 \mathrm{e}-07$ \\
\hline 16 & $2.38 \mathrm{e}-06$ & $1.05 \mathrm{e}-08$ & $6.00 \mathrm{e}-08$ & $1.99 \mathrm{e}-07$ & $2.31 \mathrm{e}-07$ & $6.23 \mathrm{e}-07$ \\
\hline 17 & $3.98 \mathrm{e}-07$ & $3.91 \mathrm{e}-07$ & $1.05 \mathrm{e}-08$ & $1.05 \mathrm{e}-08$ & $4.24 \mathrm{e}-08$ & $7.71 \mathrm{e}-07$ \\
\hline 18 & $2.43 \mathrm{e}-07$ & $1.14 \mathrm{e}-06$ & $1.05 \mathrm{e}-08$ & $1.90 \mathrm{e}-08$ & $1.05 \mathrm{e}-08$ & $2.06 \mathrm{e}-06$ \\
\hline 19 & $3.98 \mathrm{e}-07$ & $5.82 \mathrm{e}-06$ & $1.05 \mathrm{e}-08$ & $1.05 \mathrm{e}-08$ & $4.24 \mathrm{e}-08$ & $9.78 \mathrm{e}-06$ \\
\hline 20 & $1.87 \mathrm{e}-05$ & $1.05 \mathrm{e}-08$ & $1.05 \mathrm{e}-08$ & $1.05 \mathrm{e}-08$ & $2.38 \mathrm{e}-06$ & $1.03 \mathrm{e}-07$ \\
\hline 21 & $3.81 \mathrm{e}-05$ & $1.05 \mathrm{e}-08$ & $1.02 \mathrm{e}-06$ & $1.05 \mathrm{e}-08$ & $4.22 \mathrm{e}-06$ & $7.43 \mathrm{e}-06$ \\
\hline 22 & $9.52 \mathrm{e}-05$ & $1.05 \mathrm{e}-08$ & $1.05 \mathrm{e}-08$ & $1.05 \mathrm{e}-08$ & $1.22 \mathrm{e}-05$ & $1.03 \mathrm{e}-07$ \\
\hline 23 & $1.53 \mathrm{e}-06$ & $1.75 \mathrm{e}-05$ & $5.11 \mathrm{e}-08$ & $4.46 \mathrm{e}-07$ & $1.05 \mathrm{e}-08$ & $3.05 \mathrm{e}-05$ \\
\hline 24 & $2.33 \mathrm{e}-07$ & $5.79 \mathrm{e}-06$ & $1.05 \mathrm{e}-08$ & $1.05 \mathrm{e}-08$ & $1.05 \mathrm{e}-08$ & $9.73 \mathrm{e}-06$ \\
\hline Min & $2.33 \mathrm{e}-07$ & $1.05 \mathrm{e}-08$ & $1.05 \mathrm{e}-08$ & $1.05 \mathrm{e}-08$ & $1.05 \mathrm{e}-08$ & $1.03 \mathrm{e}-07$ \\
\hline
\end{tabular}

Table 8. Efficiencies of system, processes and stages calculated from the proposed method for the 24 nonlife insurance companies.

\begin{tabular}{|c|c|c|c|c|c|}
\hline No. & SYS & Process 1 & Process 2 & Stage 1 & Stage 2 \\
\hline 1 & $0.477(5)$ & $0.618(8)$ & $0.711(3)$ & $0.646(8)$ & $0.738(4)$ \\
\hline 2 & $0.301(9)$ & $0.433(18)$ & $0.625(5)$ & $0.458(19)$ & $0.657(6)$ \\
\hline 3 & $0.473(6)$ & $0.426(19)$ & $1.000(1)$ & $0.473(17)$ & $1.000(1)$ \\
\hline 4 & $0.145(22)$ & $0.286(23)$ & $0.423(10)$ & $0.317(23)$ & $0.456(12)$ \\
\hline 5 & $0.546(2)$ & $0.596(11)$ & $0.847(2)$ & $0.628(11)$ & $0.869(2)$ \\
\hline 6 & $0.332(8)$ & $0.832(3)$ & $0.308(15)$ & $0.858(3)$ & $0.387(16)$ \\
\hline 7 & $0.193(17)$ & $0.421(20)$ & $0.327(14)$ & $0.454(20)$ & $0.424(15)$ \\
\hline 8 & $0.221(15)$ & $0.533(13)$ & $0.278(17)$ & $0.572(13)$ & $0.386(17)$ \\
\hline 9 & $0.161(21)$ & $0.616(9)$ & $0.192(20)$ & $0.642(9)$ & $0.250(22)$ \\
\hline 10 & $0.237(13)$ & $0.359(22)$ & $0.548(6)$ & $0.387(22)$ & $0.613(8)$ \\
\hline 11 & $0.098(23)$ & $0.623(7)$ & $0.018(24)$ & $0.667(6)$ & $0.147(24)$ \\
\hline 12 & $0.618(1)$ & $0.835(2)$ & $0.684(4)$ & $0.860(2)$ & $0.718(5)$ \\
\hline 13 & $0.175(19)$ & $0.601(10)$ & $0.127(23)$ & $0.632(10)$ & $0.278(21)$ \\
\hline 14 & $0.200(16)$ & $0.420(21)$ & $0.355(13)$ & $0.454(20)$ & $0.440(13)$ \\
\hline 15 & $0.530(3)$ & $1.000(1)$ & $0.411(11)$ & $1.000(1)$ & $0.530(11)$ \\
\hline
\end{tabular}




\begin{tabular}{|c|c|c|c|c|c|}
\hline 16 & $0.269(11)$ & $0.741(4)$ & $0.271(18)$ & $0.771(4)$ & $0.348(19)$ \\
\hline 17 & $0.266(12)$ & $0.462(16)$ & $0.388(12)$ & $0.492(16)$ & $0.540(9)$ \\
\hline 18 & $0.178(18)$ & $0.435(17)$ & $0.301(16)$ & $0.468(18)$ & $0.381(18)$ \\
\hline 19 & $0.232(14)$ & $0.527(14)$ & $0.260(19)$ & $0.545(14)$ & $0.427(14)$ \\
\hline 20 & $0.461(7)$ & $0.674(5)$ & $0.442(9)$ & $0.709(5)$ & $0.651(7)$ \\
\hline 21 & $0.174(20)$ & $0.544(12)$ & $0.183(21)$ & $0.601(12)$ & $0.289(20)$ \\
\hline 22 & $0.493(4)$ & $0.635(6)$ & $0.501(8)$ & $0.658(7)$ & $0.750(3)$ \\
\hline 23 & $0.273(10)$ & $0.467(15)$ & $0.536(7)$ & $0.509(15)$ & $0.536(10)$ \\
\hline 24 & $0.057(24)$ & $0.241(24)$ & $0.131(22)$ & $0.264(24)$ & $0.217(23)$ \\
\hline
\end{tabular}

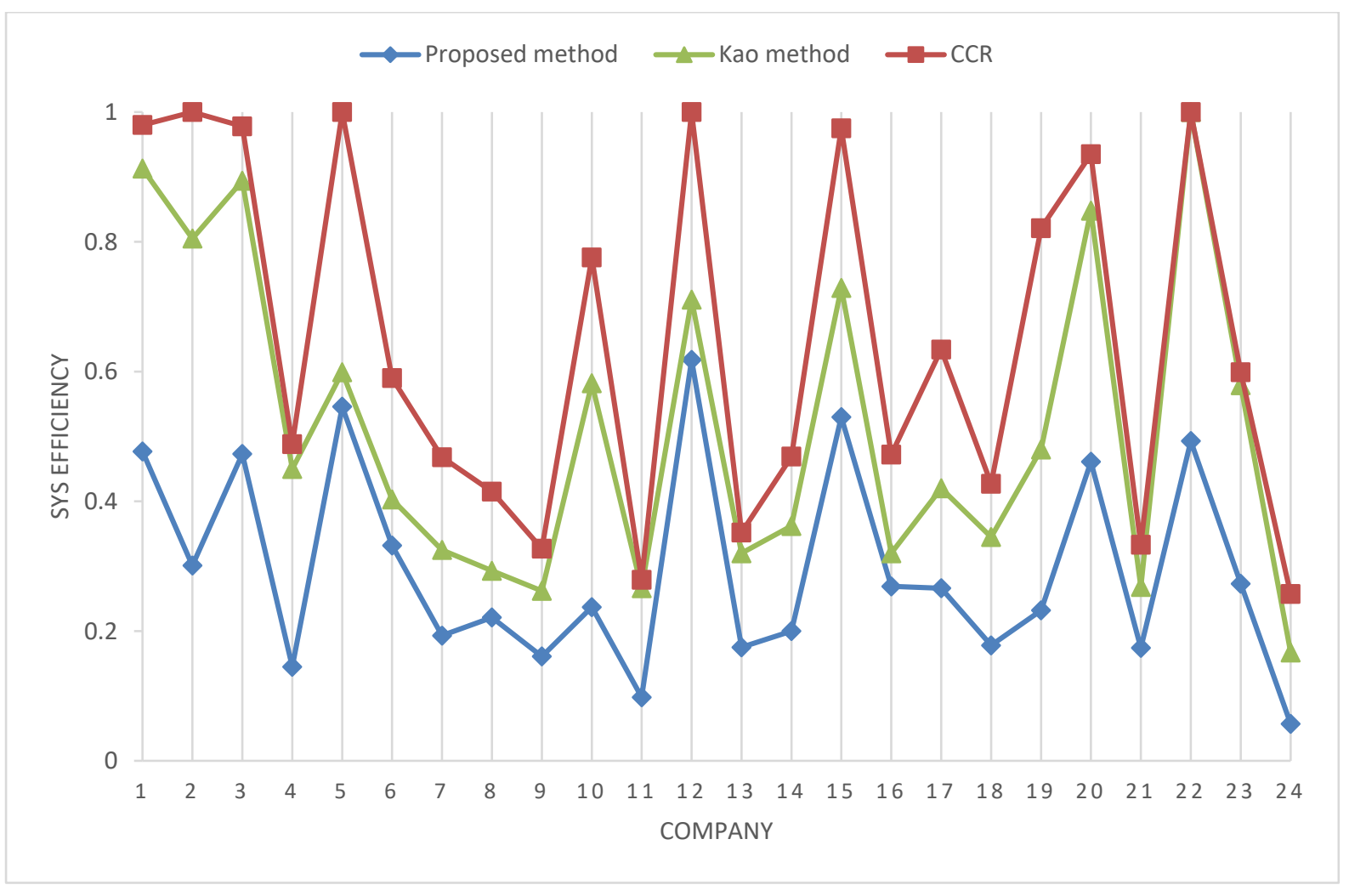

Figure 6. Comparison of results in terms of system efficiency

From the system assessment viewpoint, Figure 6 enables us to compare the results of our proposed method to those calculated from the CCR and Kao models. It becomes apparent that although the system efficiencies of the proposed method invariably result in smaller efficiency score for every company, there are the perfect correlation between the proposed method and CCR, i.e., 0.89, and the proposed method and Kao methods, i.e., 0.82 .

Companies \{Chung Hua (No. 2), Fubon (No. 5), Union (No. 12), Asia (No. 22)\} are efficient in the basic black-box CCR model and Asia (No. 22) is efficient in the network model of Kao [24]. Though no company is rated $100 \%$ efficient based on the proposed common-weights 
model, Union (No. 12) is superior to other companies. It is therefore viewed that the defining common weights for the network structures increases the discriminatory power of the model against the conventional models in the literature. Alternative point should be emphasised is that Mitsui Sumitomo (No. 24) has the most inferior performance all the time among the non-life insurance companies regardless of methods used in this application. Given the rankings of each pair of the basic CCR, Kao [24] and proposed common-weights models from the system viewpoint (see the $2^{\text {nd }}-3^{\text {rd }}$ columns of Table 6 and the $2^{\text {nd }}$ column of Table 8), Spearman's rank correlation test shows that the correlation between each pair of three models are high, where the Spearman coefficients for (CCR and Kao), (CCR and common-weights) and (Kao and common-weights) are $0.9414,0.9011$ and 0.8315 , respectively.

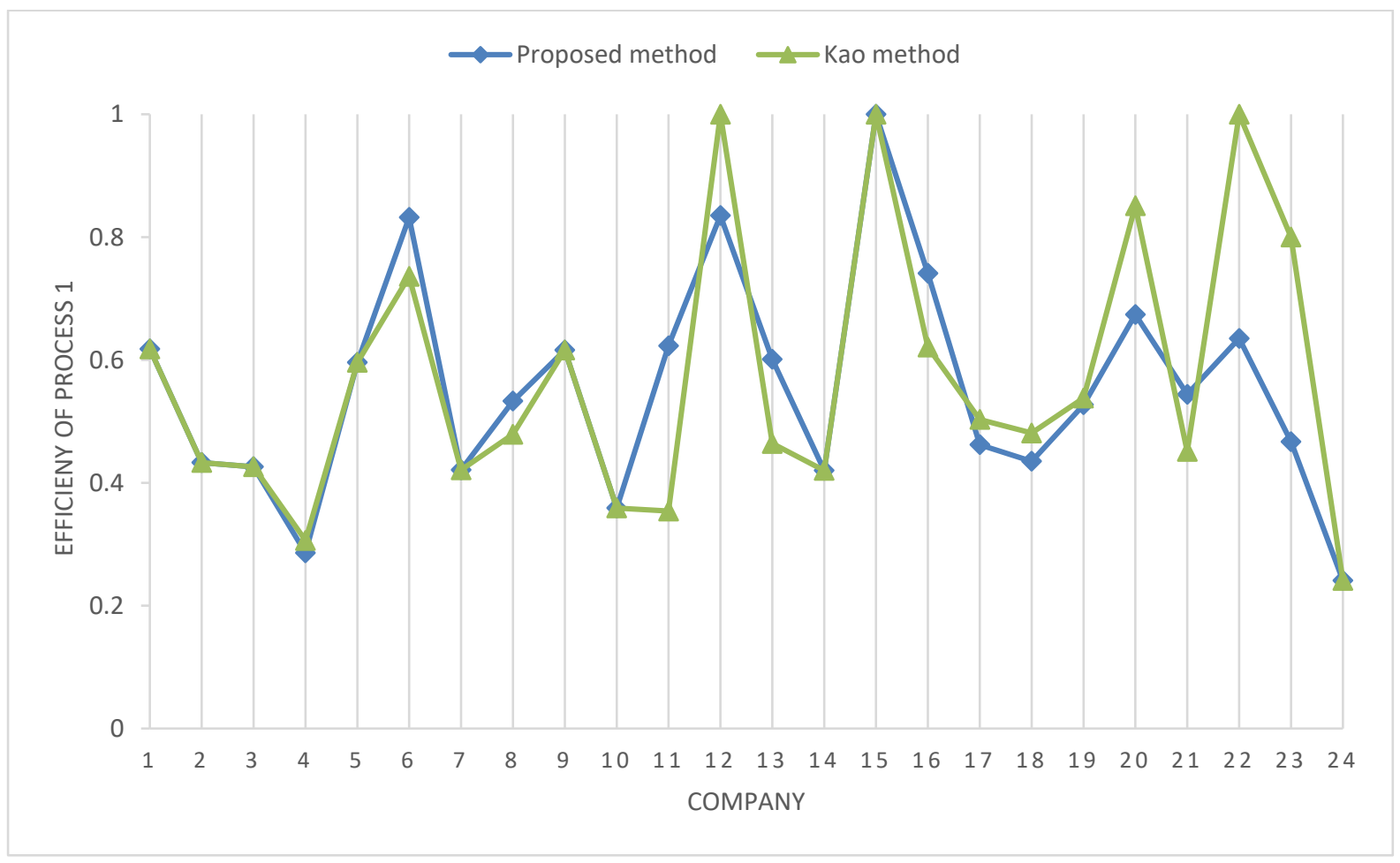

Figure 7. Comparison of results in terms of process 1's efficiencies 


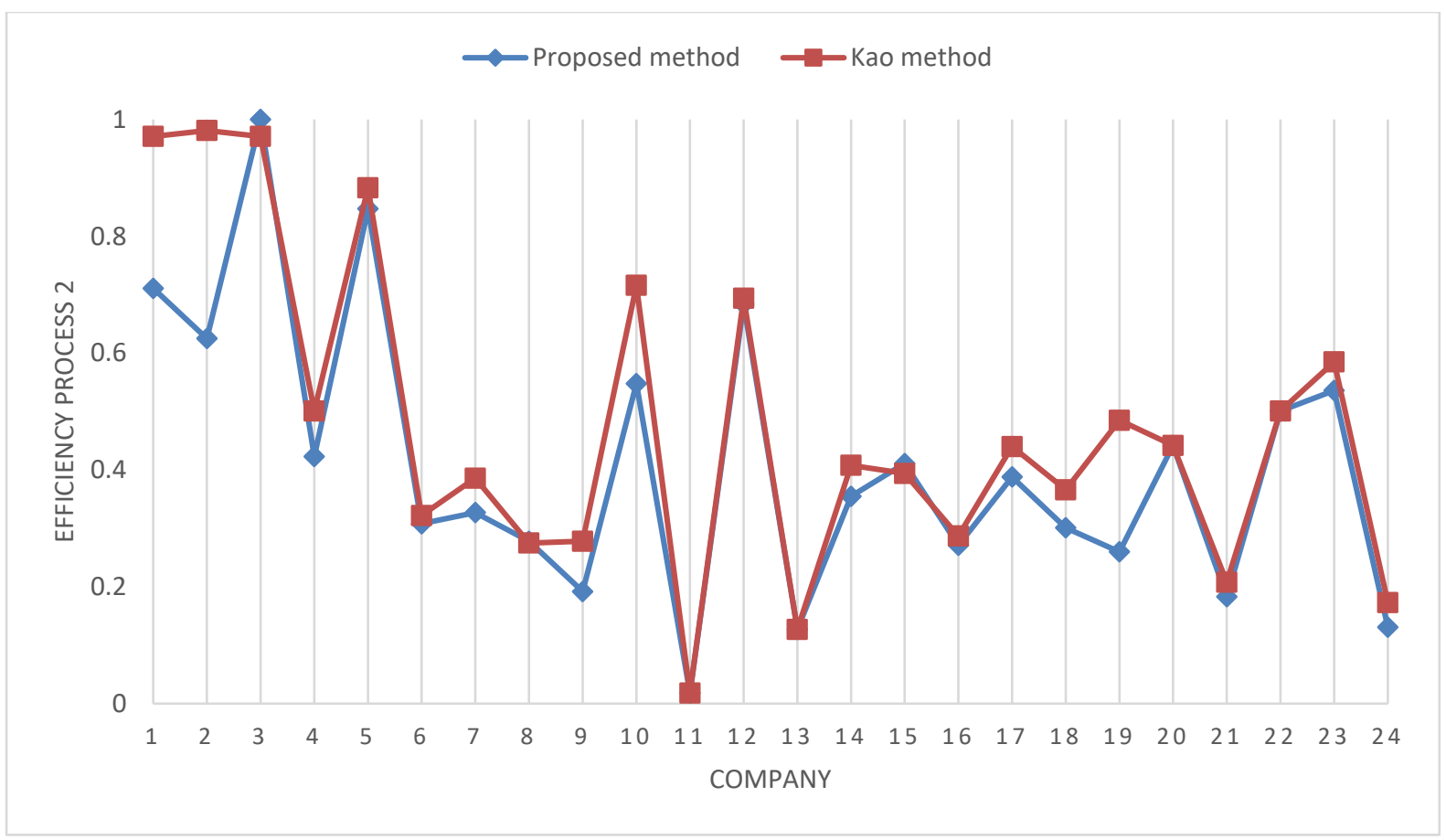

Figure 8. Comparison of results in terms of process 2's efficiencies

From the process assessment viewpoint, Figures 7 and 8 exhibit the efficiencies associated to process 1 and process 2 for every company. Apart from Companies \{No. 11, No.22, No.23\}, the efficiencies of process 1 calculated from our proposed method and those obtained from the Kao method are almost identical. On the other hand, the efficiencies of process 2 derived from the Kao method are almost the same as those attained from the proposed method in this study. Resultantly, the corresponding correlation coefficient is 0.79 (0.94) implying a perfect relationship between the efficiency measures of process 1 (2) obtained from our developed method and the efficiency measures of process 1 (2) computed from the Kao method. Minutely, Kao [24]'s results reveal that Process 1 (insurance service) for Companies \{Union (No. 12), Cathay Century (No. 15), Asia (No. 22)\} is efficient, and there is no efficient company regarding process 2 (capital investment) but Taiwan Fire (No. 1) and Tai Ping (No. 3) have the highest efficiency measures (0.971). Given the results from our method (see Table 7), process 1 for Cathay Century (No. 15) and process 2 for Tai Ping (No. 3) perform efficiently, enabling us to demonstrate the validity of the proposed method with higher discriminatory power.

From the stage assessment viewpoint, $63 \%$ of companies has better performance in stage 1 as opposed to stage 2 according to the results calculated from both proposed common-weights and Kao models. Figures 9 and 10 graphically present and compare the results of the Kao and our proposed methods for stage 1 and stage 2, respectively. It becomes apparent that for many 
companies, the efficiency measures of the Kao method are greater than those obtained from our method. We also calculate the correlation coefficient between these two methods for stage 1 (stage 2), and find a value of e-0.09 (0.74), culminating in no relationship (high) relationship between them.

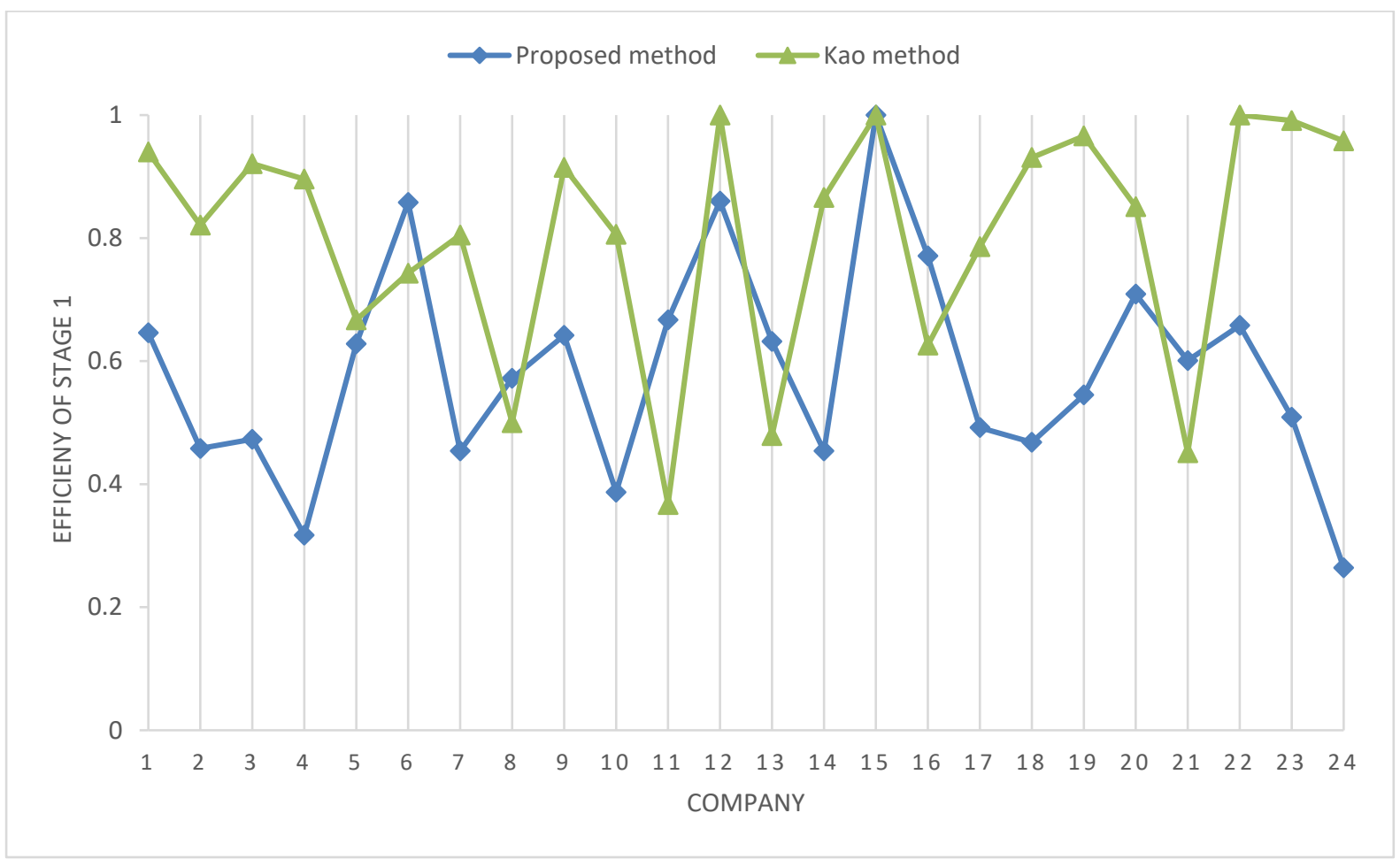

Figure 9. Comparison of results in terms of stage 1's efficiencies

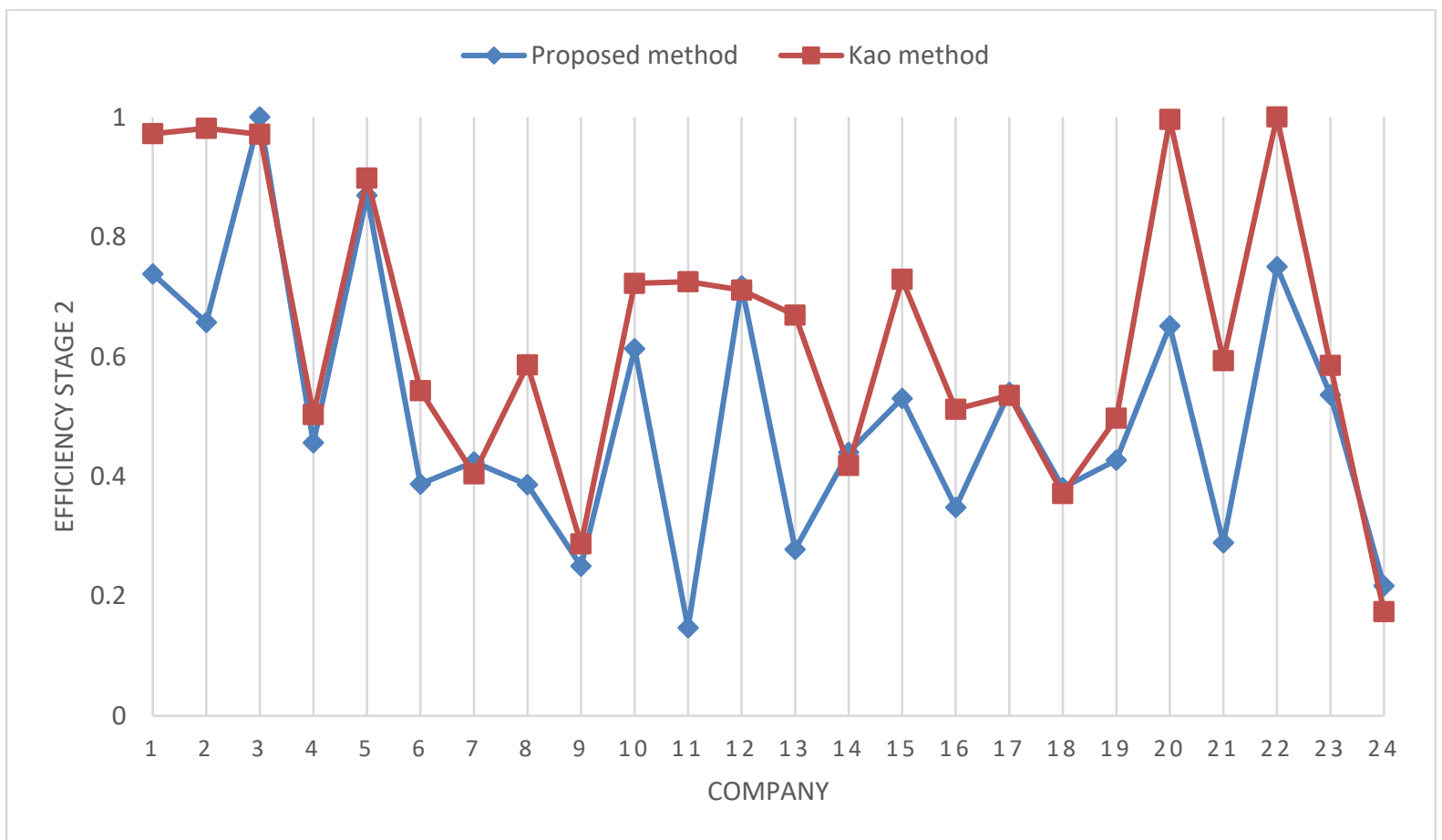

Figure 10. Comparison of results in terms of stage 2's efficiencies. 
In the view of ranking results, let us identify the Spearman's rank coefficients between each pair of system, process 1 , process 2, stage 1 and stage 2 for Kao [24] and proposed commonweights models as reported in Table 9. Note that in Table 9 the values above the diagonal correspond to the proposed common-weights model and below the diagonal correspond to the Kao model. As can be seen, the Spearman coefficient of $0.7774(0.8680)$ and $0.8252(0.7315)$ corroborates a strong correlation between the rankings of system and process 2 , and between system and stage 2 in the proposed common-weights model (Kao model). Interestingly, the rankings of both stage 1 and process 1 , and stage 2 and process 2 are highly correlated in the proposed common-weights models (see Table 9).

Table 9. The Spearman correlations for Kao and proposed method.

\begin{tabular}{|c|c|c|c|c|c|}
\hline & System & Process 1 & Process 2 & Stage 1 & Stage 2 \\
\hline System & - & 0.5070 & 0.7774 & 0.5040 & 0.8252 \\
\hline Process 1 & 0.4579 & - & $\mathrm{e}-0.032174$ & 0.9963 & 0.03391 \\
\hline Process 2 & 0.8680 & 0.2016 & - & $\mathrm{e}-0.0291$ & 0.9565 \\
\hline Stage 1 & 0.5136 & 0.3981 & 0.4593 & - & 0.03566 \\
\hline Stage 2 & 0.7315 & 0.3420 & 0.5011 & 0.0139 & - \\
\hline
\end{tabular}

\section{Conclusions}

Benchmarking is a useful tool for the performance evaluation against best practices within a group of decision-making units (DMUs) aiming at determining the inefficient areas and endeavouring to improve duly performance. The study of internal structure in benchmarking yields better insight into the evaluation organisations rather than black-box view. Regardless a large number of multiplier DEA models developed recently in the literature as to evaluate the efficiency of network production systems, much less attention is paid to the weights (multipliers) which play a crucial role in an economic or preferential sense. This paper presents a common-weights DEA model, taking account of the interrelationship of the processes within the system, to gauge the technical efficiency of whole process (system) and sub-processes. The proposed aggregate network model leads to the most favourable common weights for the purpose of calculating the efficiency of all production systems and their processes at one time. The common weights utilised for the evaluation are in line with maximising the efficiency of all systems and processes simultaneously. While the existence of multiple solutions is a quite possible problem through common-weights DEA models, the proposed model in this research yields a unique optimal solution, which is more reliable and accurate in measuring the 
efficiencies. In addition, the network system is decomposed into a series system to construct a relational network DEA model. Finally, this paper presents one simple numerical example and one case study of the non-life insurance companies to illustrate the applicability of the proposed common-weights network model.

The developed framework in this research can potentially lend itself to many practical applications such as supply chain performance evaluation. An important topic for future research could extend the formulation to include fuzzy, interval or stochastic data to deal with inherent uncertainty in real evaluation problems.

\section{Conflict of interest}

No potential conflict of interest exists for this study.

\section{References}

[1] Charnes, A, Cooper, WW, Rhodes, E (1978) Measuring the efficiency of decision making units. Eur J Oper Res 2, 429-444.

[2] Banker, RD., Charnes, A., Cooper, WW, (1984) Some models for estimating technical and scale efficiencies in data envelopment analysis. Manage Sci 30, 1078-1092.

[3] Charnes A, Cooper WW, Lewin AY, Seiford LM (1994) Data envelopment analysis: theory, methodology, and applications. Norwell: Kluwer Academic Publishers.

[4] Cook, WD, Roll, Y, Kazakov, A (1990) A DEA model for measuring the relative efficiency of highway maintenance patrols. INFOR: Information Systems and Operational Research, 28(2), 113-124.

[5] Roll, Y, Cook, WD, Golany, B (1991) Controlling factor weights in data envelopment analysis. IIE transactions, 23(1), 2-9.

[6] Doyle, JR, Green, RH (1995) Cross-evaluation in DEA: Improving discrimination among DMUs. INFOR: Information Systems and Operational Research, 33(3), 205-222.

[7] Liu, FHF, Peng, HH (2008) Ranking of units on the DEA frontier with common weights. Comput Oper Res 35(5), 1624-1637.

[8] Saati, S, Hatami-Marbini, A, Agrell, PJ, Tavana, M (2012) A common set of weight approach using an ideal decision making unit in data envelopment analysis. J Ind Manag Optim 8(3), 623-637.

[9] Hosseinzadeh Lotfi, F, Hatami-Marbini, A, Agrell, PJ, Aghayi, N, Gholami, K (2013) Allocating fixed resources and setting targets using a common-weights DEA approach. Comput Ind Eng 64(2), 631-640. 
[10] Hatami-Marbini, A, Tavana, M, Agrell, PJ, Hosseinzadeh Lotfi, F, Ghelej Beigi, Z (2015) A common-weights DEA model for centralized resource reduction and target setting. Comput Ind Eng 79, 195-203.

[11] Wang, YM, Chin, KS (2010) A neutral DEA model for cross-efficiency evaluation and its extension. Expert Syst Appl 37(5), 3666-3675.

[12] Färe, R, Grosskopf, S (1996) Intertemporal production frontiers: With dynamic DEA. Kluwer Academic Publishers.

[13] Färe, R, Grosskopf, S (2000) Network DEA. Socio Econ Plan Sci, 34, 35-49.

[14] Wang, CH, Gopal, R, Zionts, S (1997) Use of data envelopment analysis in assessing information technology impact on firm performance. Ann Oper Res 73, 191-213.

[15] Seiford, L, Zhu, J (1999) Profitability and marketability of the top 55 US commercial banks. Manage Sci, 45(9), 1270-1288.

[16] Chen, Y, Zhu, J (2004) Measuring information technology's indirect impact on firm performance. Inform Technol Manage, 5(12), 9-22.

[17] Tone, K, Tsutsui, M (2009) Network DEA: A slacks-based measure approach. Eur J Oper Res 197(1), 243-252.

[18] Castelli, L, Pesenti, R, Ukovich, W (2010) A classification of DEA models when the internal structure of the decision-making units is considered. Ann Oper Res 173(1), 207-235.

[19] Cook, WD, Liang, L, Zhu, J (2010). Measuring performance of two-stage network structures by DEA: A review and future perspective. Omega, 38(6), 423-430.

[20] Agrell, PJ, Hatami-Marbini, A (2013) Frontier-based performance analysis models for supply chain management: State of the art and research directions. Comput Ind Eng 66(3), 567-583.

[21] Kao, C (2014) Network data envelopment analysis: A review. Eur J Oper Res 239(1), 116.

[22] Castelli, L, Pesenti, R (2014) Network, shared flow and multi-level DEA models: A critical review. In data envelopment analysis (pp. 329-376). Springer US.

[23] Kao, C, Hwang, SN (2008) Efficiency decomposition in two-stage data envelopment analysis: An application to non-life insurance companies in Taiwan. Eur J Oper Res 185(1), 418-429.

[24] Kao, C (2009) Efficiency decomposition in network data envelopment analysis: A relational model. Eur J Oper Res 192(3), 949-962. 
[25] Yang, C, Liu, HM (2012) Managerial efficiency in Taiwan bank branches: A network DEA. Econ Model 29(2), 450-461.

[26] Zimmermann, HJ, (1978) Fuzzy programming and linear programming with several objective functions. Fuzzy Set Syst 1, 45-55.

[27] DeWispelare, AR, Sage, AP (1981) On combined multiple objective optimization theory and multiple attribute utility theory for evaluation and choice making. Large Scale Syst Inform Decision Technol 2, 1-19.

[28] Charnes, A, Cooper, WW (1977) Goal programming and multiple objective optimizations: Part 1. Eur J Oper Res 1(1), 39-54.

[29] Sakawa, M (1982) Interactive multiobjective decision making by the sequential proxy optimization technique: SPOT. Eur J Oper Res 9(4), 386-396.

[30] Sakawa, M (1993) Fuzzy Sets and Interactive Multiobjective Optimization. Plenum Press, New York.

[31] Toloo, M (2014) An epsilon-free approach for finding the most efficient unit in DEA. Appl Mathl Model, 38(13), 3182-3192.

[32] Fecher, F, Kessler, D, Perelman, S, Pestieau, P (1993) Productive performance of the French insurance industry. J Prod anal 4(1-2), 77-93. 


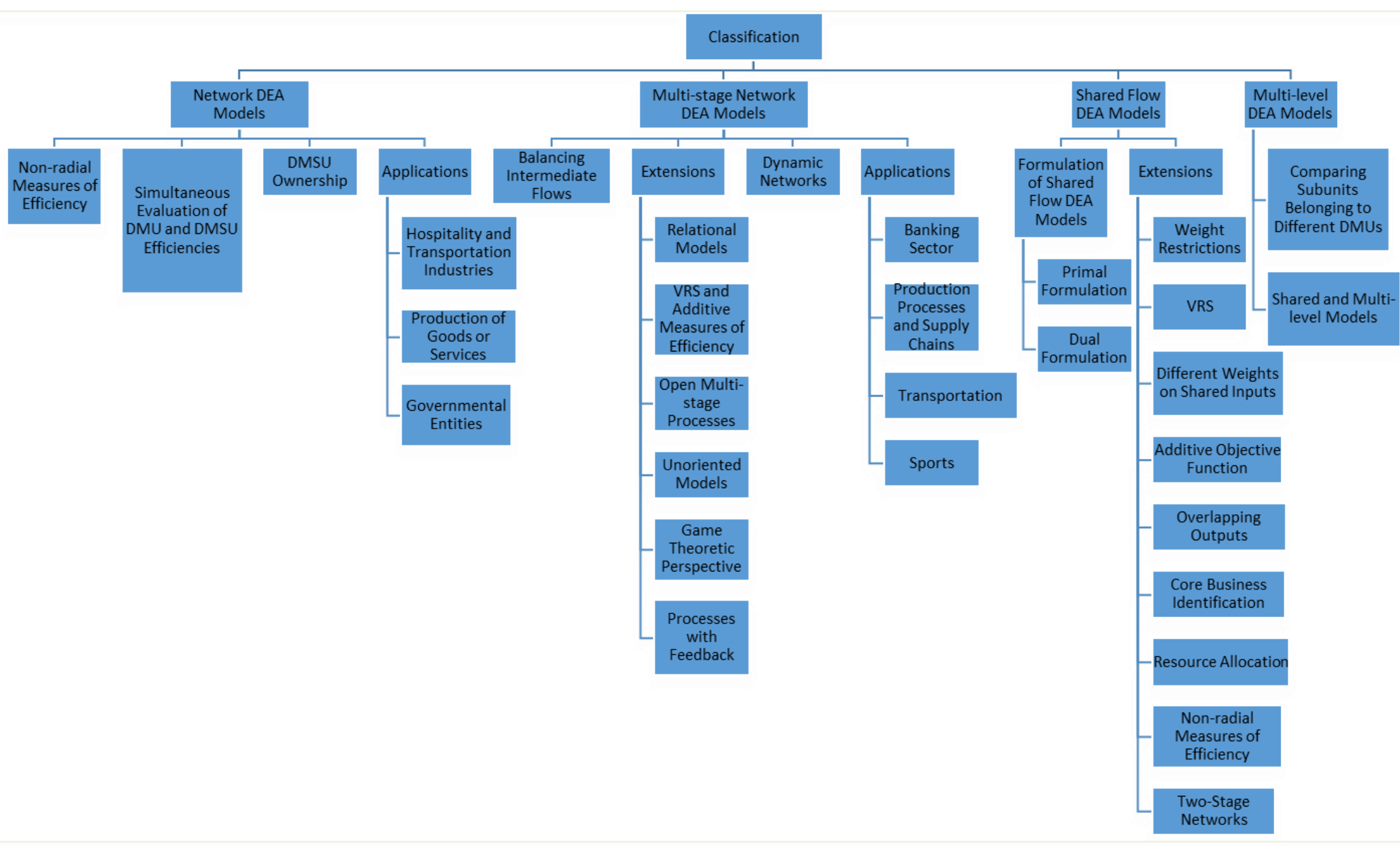

Figure 1. A classification of network models 